\title{
Colored Vertex Models, Colored IRF Models and Invariants of Trivalent Colored Graphs
}

\author{
Tetsuo Deguchi and Yasuhiro Akutsu ${ }^{\dagger}$ \\ Department of Physics, Faculty of Science, \\ University of Tokyo, Hongo, Bunkyo-ku, Tokyo 113, Japan \\ $\dagger$ Department of Physics, Faculty of Science \\ Osaka University, Machikaneyama-cho 1-1, Toyonaka 560, Japan
}

\begin{abstract}
.
We present formulas for the Clebsch-Gordan coefficients and the Racah coefficients for color representations ( $N$-dimensional representations with $\left.q^{2 N}=1\right)$ of $U_{q}(s l(2))$. We discuss colored vertex models and colored IRF (Interaction Round a Face) models from the color representations of $U_{q}(s l(2))$. We construct invariants of trivalent colored oriented framed graphs from color representations of $U_{q}(s l(2))$.
\end{abstract}




\section{Introduction}

Recently a new hierarchy of $N$-state colored braid matrices $(N=2,3, \cdots)$ has been constructed which have color variables $\alpha$ and $\beta$. [1, 2, 3, 国 We regard colored braid as a braid on strings with colors. From the colored braid matrices a new series of isotopy invariants of colored links generalizing the multivariable Alexander polynomial has been obtained. [5] Using the limit $q^{2} \rightarrow \omega$, we have derived the $N$-state colored braid matrices from infinite dimensional representations of $U_{q}(s l(2))$. [6] Here $\omega$ is a primitive $N$-th root of unity. Thus the colored braid matrices are derived from the $N$-dimensional representations [7] of $U_{q}(s l(2))$ with $q^{2 N}=1$. We call the $N$ dimensional representation as (finite dimsional) color representation. We call infinite dimensional representation with a free parameter infinite dimensional color representation. We note that representation theory of quantum groups with $q$ roots of unity is given in Refs. [8, 9].

In this paper we present formulas for the Clebsch-Gordan coefficients and the Racah coefficients of the color representations of $U_{q}(\operatorname{sl}(2))$. We introduce colored IRF models and colored vertex models from the color representations of $U_{q}(\hat{s} l(2))$. We construct invariants of trivalent colored oriented graphs from the Clebsch-Gordan coefficients of the color representations. The new graph invariants give generalizations of the multivariable Alexander polynomial of links.

There are various viewpoints associated with the colored vertex models. The $N=2$ case of the colored vertex model corresponds to the trigonometric limit of the Felderhof's solution [10] of the free fermion model. [11] The non-colored case of the trigonometric limit is related to the Lie superalgebra $\operatorname{gl}(1 \mid 1)(\operatorname{sl}(1 \mid 1))$. [12] [13, 14, 15] It has been pointed out [16] that the colored case of the trigonometric limit is related to $U_{q}(g l(1 \mid 1))$. From the 
different viewpoint it has also been shown [17] that the colored case of the trigonometric limit is related to $U_{q}(\operatorname{sl}(2))$.

The free fermion model and the colored vertex models are related to the link polynomials, which vanish for disconnected links. From the $g l(m \mid m)$ model we have the Alexander polynomial. [12] A state model for the multivariable Alexander polynomial was constructed from the trigonometric limit of the free fermion model. [18] Several examples of braid matrices with the vanishing property were calculated by Lee, Couture and Schmeing, by directly solving the braid relation up to the 6 state case. [19]

The outline of this paper is given in the following. In $\S 2$ we give the Clebsch-Gordan coefficents and the Racah coefficients of infinite dimensional representations of $U(\operatorname{sl}(2))$. In $\S 3$ we present formulas for the Clebsch-Gordan coefficients and the Racah coefficients for infinite-dimensional color representations of $U_{q}(\operatorname{sl}(2))$. By taking the limit $q^{2} \rightarrow \omega$ we derive formulas for the Clebsch-Gordan coefficients and the Racah coefficients of finite-dimensional color representations of $U_{q}(\operatorname{sl}(2))$. In $\S 4$ we discuss the colored vertex models from the viewpoint of color representations of the $U_{q}(\hat{s} l(2))$. In $\S 5$ we construct IRF models associated with color representations of $U_{q}(\operatorname{sl}(2))$, which we have called colored IRF models. In $\S 6$ we obtain new invariants of trivalent colored graphs from the Clebsch-Gordan coefficients of color representations.

\section{CGC of infinte dimensional representations of $U(\operatorname{sl}(2))$}

Let us discuss representations of the universal enveloping algebra $U(\operatorname{sl}(2))$. The defining relations of the algebra $U(s l(2))$ are given by the following.

$$
\left[H, X^{ \pm}\right]= \pm 2 X^{ \pm}
$$




$$
\left[X^{+}, X^{-}\right]=H .
$$

The comultiplication is given by

$$
\begin{aligned}
\Delta\left(X^{ \pm}\right) & =X^{ \pm} \otimes I+I \otimes X^{ \pm} \\
\Delta(H) & =H \otimes I+I \otimes H .
\end{aligned}
$$

Let us define infinite dimensional representation $\left(\pi^{p}, V^{(p)}\right)$ of $s l(2)$. Let $V^{(p)}$ be an infinite dimensional vector space over $\mathbf{C}$ with basis $e_{0}, e_{1}, \cdots$, where $e_{a}$ is a basis vector in $V^{(p)}$ with the property $\left(e_{a}\right)_{b}=\delta_{b a}$. We define the matrix elements of the generators as follows.

$$
\begin{aligned}
\left(\pi^{p}\left(X^{+}\right)\right)_{b}^{a} & =\sqrt{(2 p-a)(a+1)} \cdot \delta_{a+1, b}, \\
\left(\pi^{p}\left(X^{-}\right)\right)_{b}^{a} & =\sqrt{a(2 p-a+1)} \cdot \delta_{a-1, b}, \\
\left(\pi^{p}(H)\right)_{b}^{a} & =(2 p-2 a) \cdot \delta_{a, b} .
\end{aligned}
$$

The variable $a$ takes a nonnegative integer; $a=0,1, \cdots$. The basis vectors for the infinite dimensional representation are labeled by $(p, z)$, where $p \in \mathbf{C}$ and $z \in \mathbf{Z}_{\geq 0}$. Here the symbol $\mathbf{Z}_{\geq 0}$ denotes the set of nonnegative integers. We write the representation $\left(\pi^{p}, V^{(p)}\right)$ simply by $V^{(p)}$, and the basis vector $e_{z}$ by $\mid p, z>$. In terms of the basis vector the representation (2.3) is expressed as follows.

$$
\begin{aligned}
X^{+} \mid p, z> & =\sqrt{(2 p-z+1) z} \mid p, z-1>, \\
X^{-} \mid p, z> & =\sqrt{(2 p-z)(z+1)} \mid p, z+1>, \\
H \mid p, z> & =(2 p-2 z) \mid p, z>.
\end{aligned}
$$

Let us discuss decomposition of the tensor product $V^{\left(p_{1}\right)} \otimes V^{\left(p_{2}\right)}$. We assume the following

$$
\left|p_{1}, p_{2} ; p_{3}, z_{3}>=\sum_{z_{1}, z_{2}} C\left(p_{1}, p_{2}, p_{3} ; z_{1}, z_{2}, z_{3}\right)\right| p_{1}, z_{1}>\otimes \mid p_{2}, z_{2}>.
$$


From the comultiplication rule $\Delta(H)=H \otimes I+I \otimes H\left(H_{t o t}=H_{1}+H_{2}\right)$ we can show

$$
C\left(p_{1}, p_{2}, p_{3} ; z_{1}, z_{2}, z_{3}\right)=0 \text {, unless } p_{1}-z_{1}+p_{2}-z_{2}=p_{3}-z_{3}
$$

The relation (2.6) gives the charge conservation law. Using (2.6) and the condition $z_{i} \in \mathbf{Z}_{\geq 0}$, we can show

$$
C\left(p_{1}, p_{2}, p_{3} ; z_{1}, z_{2}, z_{3}\right)=0 \text {, unless } p_{3}=p_{1}+p_{2}-n, n \in \mathbf{Z}_{\geq 0} \text {. }
$$

Thus we have for $n \in \mathbf{Z}_{\geq 0}$

$$
\begin{aligned}
& \mid p_{1}, p_{2} ; p_{1}+p_{2}-n, z_{3}> \\
= & \sum_{z_{1}=0}^{z_{3}+n} C\left(p_{1}, p_{2}, p_{1}+p_{2}-n ; z_{1}, z_{3}+n-z_{1}, z_{3}\right)\left|p_{1}, z_{1}>\otimes\right| p_{2}, z_{3}+n-z_{1}>.
\end{aligned}
$$

The fusion rules for the tensor product $V^{\left(p_{1}\right)} \otimes V^{\left(p_{2}\right)}$ are summarized as follows.

$$
V^{\left(p_{1}\right)} \otimes V^{\left(p_{2}\right)}=\sum_{p_{3}} N_{p_{1} p_{2}}^{p_{3}} V^{\left(p_{3}\right)}
$$

where

$$
\begin{aligned}
N_{p_{1} p_{2}}^{p_{3}} & =1 \text { for } p_{3}=p_{1}+p_{2}-n, n \in \mathbf{Z}_{\geq 0} \\
& =0, \quad \text { otherwise. }
\end{aligned}
$$

We assume the following convention of the phase factors of the CG coefficients.

$$
C\left(p_{1}, p_{2}, p_{1}+p_{2}-n ; 0, n, 0\right)=1 \text {. }
$$

Then the Clebsch-Gordan coefficients for infinite dimensional representations are given by the following.

$$
C\left(p_{1}, p_{2}, p_{1}+p_{2}-n ; z_{1}, z_{2}, z_{3}\right)=\delta\left(z_{3}, z_{1}+z_{2}-n\right)
$$




$$
\begin{aligned}
& \times \sqrt{\left(2 p_{1}+2 p_{2}-2 n+1\right)}\left(\frac{\left(2 p_{1}-n\right) !\left(2 p_{2}-n\right) !(n) !}{\left(2 p_{1}+2 p_{2}-n+1\right) !}\right)^{1 / 2} \\
& \times \sqrt{\left(2 p_{1}-z_{1}\right) !\left(2 p_{2}-z_{2}\right) !\left(2 p_{1}+2 p_{2}-2 n-z_{3}\right) !\left(z_{1}\right) !\left(z_{2}\right) !\left(z_{3}\right) !} \\
& \times \sum_{\nu} \frac{(-1)^{\nu}}{(\nu) !(n-\nu) !} \frac{1}{\left(z_{1}-\nu\right) !\left(z_{2}-n+\nu\right) !} \\
& \times \frac{1}{\left(2 p_{1}-n-z_{1}+\nu\right) !\left(2 p_{2}-z_{2}-\nu\right) !} .
\end{aligned}
$$

Here we have defined the symbol $(p)$ ! through the Gamma function

$$
(p) !=\Gamma(p+1) .
$$

The sum over the integer $\nu$ in (2.12) is taken under the following condition

$$
\max \left\{0, n-z_{2}\right\} \leq \nu \leq \min \left\{n, z_{1}\right\} .
$$

We can derive the Clebsch-Gordan coefficients (2.12) following Racah's derivation [20] (see also Appendix A).

Hereafter we assume that the symbols $p$ and $p_{j}$ denote complex parameters $\left(p, p_{j} \in \mathbf{C}\right)$, and $2 j$ and $2 j_{k}$ denote nonnegative integers $\left(2 j, 2 j_{k} \in \mathbf{Z}_{\geq 0}\right)$. We also assume that $2 \mu_{j}(j=1,2,3)$ denote either non-negative integers $\left(2 \mu_{j} \in \mathbf{Z}_{\geq 0}\right)$ or complex parameters $\left(\mu_{j} \in \mathbf{C}\right)$.

Let $V^{(j)}$ denote the representation with dimension $2 j+1$ (spin $j$ representation). Let us consider the following decomposition of the tensor product.

$$
V^{\left(\mu_{1}\right)} \otimes V^{\left(\mu_{2}\right)}=\sum_{p_{3}} N_{\mu_{1} \mu_{2}}^{p_{3}} V^{\left(p_{3}\right)}, p_{3} \in \mathbf{C} .
$$

Here we consider the two cases, (1) $\left(\mu_{1}, \mu_{2}\right)=\left(p_{1}, j_{2}\right)\left(p_{1} \in \mathbf{C}, 2 j_{2} \in \mathbf{Z}_{\geq 0}\right)$; (2) $\left(\mu_{1}, \mu_{2}\right)=\left(j_{1}, p_{2}\right)\left(2 j_{1} \in \mathbf{Z}_{\geq 0}, p_{2} \in \mathbf{C}\right)$.

Then we have the following fusion rules.

Case $(1)\left(\mu_{1}, \mu_{2}\right)=\left(p_{1}, j_{2}\right)\left(p_{1} \in \mathbf{C}, 2 j_{2} \in \mathbf{Z}_{\geq 0}\right)$;

$$
\begin{aligned}
N_{p_{1} j_{2}}^{p_{3}} & =1 \text { if } p_{3}=p_{1}+j_{2}-n, 0 \leq n \leq 2 j_{2}, n \in \mathbf{Z}_{\geq 0} \\
& =0, \quad \text { otherwise. }
\end{aligned}
$$


Case $(2)\left(\mu_{1}, \mu_{2}\right)=\left(j_{1}, p_{2}\right)\left(2 j_{1} \in \mathbf{Z}_{\geq 0}, p_{2} \in \mathbf{C}\right)$

$$
\begin{aligned}
N_{j_{1} p_{2}}^{p_{3}} & =1 \text { if } p_{3}=j_{1}+p_{2}-n, 0 \leq n \leq 2 j_{1}, n \in \mathbf{Z}_{\geq 0} \\
& =0, \quad \text { otherwise. }
\end{aligned}
$$

The Clebsch-Gordan coefficients are given by the following.

$$
\begin{aligned}
& C\left(\mu_{1}, \mu_{2}, \mu_{1}+\mu_{2}-n ; z_{1}, z_{2}, z_{3}\right)=\delta\left(z_{3}, z_{1}+z_{2}-n\right) \\
& \times \sqrt{\left(2 \mu_{1}+2 \mu_{2}-2 n+1\right)}\left(\frac{\left(\mu_{1}-n\right) !\left(2 \mu_{2}-n\right) !(n) !}{\left(2 \mu_{1}+2 \mu_{2}-n+1\right) !}\right)^{1 / 2} \\
& \times \sqrt{\left(2 \mu_{1}-z_{1}\right) !\left(2 \mu_{2}-z_{2}\right) !\left(2 \mu_{1}+2 \mu_{2}-2 n-z_{3}\right) !\left(z_{1}\right) !\left(z_{2}\right) !\left(z_{3}\right) !} \\
& \times \sum_{\nu} \frac{(-1)^{\nu}}{(\nu) !(n-\nu) !} \frac{1}{\left(z_{1}-\nu\right) !\left(z_{2}-n+\nu\right) !} \\
& \times \frac{1}{\left(2 \mu_{1}-n-z_{1}+\nu\right) !\left(2 \mu_{2}-z_{2}-\nu\right) !}
\end{aligned}
$$

Here the sum over the integer $\nu$ is taken under the following conditions:

Case (1) $\mu_{1}=p_{1}, \mu_{2}=j_{2}$,

$$
\max \left\{0, n-z_{2}\right\} \leq \nu \leq \min \left\{n, z_{1}, 2 j_{2}-z_{2}\right\}
$$

Case $(2) \mu_{1}=j_{1}, \mu_{2}=p_{2}$,

$$
\max \left\{0, n-z_{2}, n+z_{1}-2 j_{1}\right\} \leq \nu \leq \min \left\{n, z_{1}\right\}
$$

We note that the formula (2.18) can be derived from (2.12) by taking the limit $p_{2} \rightarrow j_{2}$. The branching rule (2.17) can be shown through this limiting procedure.

The Clebsch-Gordan coefficients satisfy the orthogonality relations for the cases $\left(\mu_{1}, \mu_{2}\right)=\left(p_{1}, p_{2}\right),\left(p_{1}, j_{2}\right)$, and $\left(j_{1}, p_{2}\right)$.

$$
\begin{array}{rl}
\delta_{n n^{\prime}}=\sum_{z_{1}} & C\left(\mu_{1}, \mu_{2}, \mu_{1}+\mu_{2}-n ; z_{1}, \tau-z_{1}, \tau-n\right) \\
\times & C\left(\mu_{1}, \mu_{2}, \mu_{1}+\mu_{2}-n^{\prime} ; z_{1}, \tau-z_{1}, \tau-n^{\prime}\right) .
\end{array}
$$


Let us discuss the Racah coefficients for finite and infinite dimensional representations. Using the formulas for the Clebsch-Gordan coefficients (2.12), and (2.18) we can calculate the Racah coefficients. We recall that $2 \mu_{i}$ $(i=1,2,3)$ is either a nonnegative integer $\left(2 \mu_{i} \in \mathbf{Z}_{\geq 0}\right)$ or a complex parameter $\left(\mu_{i} \in \mathbf{C}\right)$. We introduce $\mu, \mu^{\prime}, \mu^{\prime \prime}$ by

$$
\mu=\mu_{1}+\mu_{2}+\mu_{3}-n, \mu^{\prime}=\mu_{1}+\mu_{2}-n^{\prime}, \mu^{\prime \prime}=\mu_{2}+\mu_{3}-n^{\prime \prime},
$$

where $n, n^{\prime}, n^{\prime \prime} \in \mathbf{Z}_{\geq 0}$.

The Racah coefficients $W\left(\mu_{1}, \mu_{2}, \mu, \mu_{3} ; \mu^{\prime}, \mu^{\prime \prime}\right)$ are defined by 20]

$$
\begin{aligned}
& \mid \mu_{1} \mu_{2}\left(\mu^{\prime}\right) \mu_{3} \mu z>= \\
& =\sum_{\mu^{\prime \prime}} \sqrt{\left(2 \mu^{\prime}+1\right)\left(2 \mu^{\prime \prime}+1\right)} W\left(\mu_{1}, \mu_{2}, \mu, \mu_{3} ; \mu^{\prime}, \mu^{\prime \prime}\right) \mid \mu_{1}, \mu_{2} \mu_{3}\left(\mu^{\prime \prime}\right) \mu z>.
\end{aligned}
$$

We note that the Racah coeffcients do not depend on $z$ by the definition (2.23). From the definition of the Racah coefficients, we have

$$
\begin{aligned}
& C\left(\mu_{1}, \mu_{2}, \mu^{\prime} ; w_{1}, w_{2}, w_{1}+w_{2}-n^{\prime}\right) C\left(\mu^{\prime}, \mu_{3}, \mu ; w_{1}+w_{2}-n^{\prime}, w_{3}, w_{1}+w_{2}+w_{3}-n\right) \\
= & \sum_{\mu^{\prime \prime}} R_{\mu^{\prime \prime} \mu^{\prime}} C\left(\mu_{1}, \mu^{\prime \prime}, \mu ; w_{1}, w_{2}+w_{3}-n^{\prime \prime}, w_{1}+w_{2}+w_{3}-n\right) \\
& \times C\left(\mu_{2}, \mu_{3}, \mu^{\prime \prime} ; w_{2}, w_{3}, w_{2}+w_{3}-n^{\prime \prime}\right) .
\end{aligned}
$$

Here $w_{1}, w_{2}, w_{3}, \in \mathbf{Z}_{\geq 0}$. Applying the orthogonality relation (2.21) to (2.24) we have a formula for the Racah coefficients. The derivation of the Racah coefficients consists of the following procedures. (1) Let us set $w_{2}+w_{3}=z^{\prime \prime}$. (2) Then multiply both the left hand side and right hand side of (2.24) by $\sum_{w_{2}} C\left(\mu_{2}, \mu_{3}, \mu_{2}+\mu_{3}-N^{\prime \prime} ; w_{2}, z^{\prime \prime}-w_{2}, z^{\prime \prime}-N^{\prime \prime}\right)$. (3) Rewrite $N^{\prime \prime}$ by $n^{\prime \prime}$, and introduce $z=w_{1}+z^{\prime \prime}=w_{1}+w_{2}+w_{3}$. (4) Multiply both sides by $\sum_{w_{1}} C\left(\mu_{1}, \mu_{2}+\mu_{3}-n^{\prime \prime}, \mu_{1}+\mu_{2}+\mu_{3}-n ; w_{1}, z-w_{1}, z+n^{\prime \prime}-n\right)$. 
Thus we have the Racah coefficients $W\left(\mu_{1}, \mu_{2}, \mu, \mu_{3} ; \mu^{\prime}, \mu^{\prime \prime}\right)$ as follows.

$$
\begin{aligned}
R_{\mu^{\prime \prime} \mu^{\prime}}= & \sqrt{\left(2 \mu^{\prime}+1\right)\left(2 \mu^{\prime \prime}+1\right)} W\left(\mu_{1}, \mu_{2}, \mu, \mu_{3} ; \mu^{\prime}, \mu^{\prime \prime}\right) \\
= & \sum_{w_{1}} \sum_{w_{2}} C\left(\mu_{1}, \mu_{2}, \mu^{\prime} ; w_{1}, w_{2}, w_{1}+w_{2}-n^{\prime}\right) \\
& \times C\left(\mu^{\prime}, \mu_{3}, \mu ; w_{1}+w_{2}-n^{\prime}, z-w_{1}-w_{2}, z-n\right) \\
& \times C\left(\mu_{2}, \mu_{3}, \mu_{2}+\mu_{3}-n^{\prime \prime} ; w_{2}, z-w_{1}-w_{2}, z-w_{1}-n^{\prime \prime}\right) \\
& \times C\left(\mu_{1}, \mu^{\prime \prime}, \mu ; w_{1}, z-w_{1}, z+n^{\prime \prime}-n\right) .
\end{aligned}
$$

\section{CGC of color representations of $U_{q}(s l(2))$}

\subsection{Infinite dimensional case}

The generators of the algebra are $\left\{X^{+}, X^{-}, H\right\}$ with the defining relations [21, 22]

$$
\begin{aligned}
{\left[H, X^{ \pm}\right] } & = \pm 2 X^{ \pm} \\
{\left[X^{+}, X^{-}\right] } & =\frac{q^{H}-q^{-H}}{q-q^{-1}} .
\end{aligned}
$$

The comultiplication is given by

$$
\begin{aligned}
\Delta(H) & =H \otimes I+I \otimes H, \\
\Delta\left(X^{ \pm}\right) & =X^{ \pm} \otimes q^{H / 2}+q^{-H / 2} \otimes X^{ \pm} .
\end{aligned}
$$

We use the following symbols for $q$-analogs.

$$
[n]_{q}=\frac{q^{n}-q^{-n}}{q-q^{-1}}, \quad[n]_{q} !=\prod_{k=1}^{n}[k]_{q}, \quad[p ; n]_{q} !=\prod_{k=0}^{n-1}[p-k]_{q} .
$$

Here $n$ is a positive integer. For the case $n=0$ we assume

$$
[0]_{q} !=[p ; 0]_{q} !=1
$$


Let us define an infinite dimensional representation $\left(\pi_{q}^{p}, V^{(p)}\right)$ of $U_{q}(\operatorname{sl}(2))$. We recall that $p$ is a complex parameter. Let $V^{(p)}$ be an infinite dimensional vector space over $\mathbf{C}$ with basis $e_{0}, e_{1}, \cdots$, where $e_{a}$ is a basis vector in $V^{(p)}$ with the property $\left(e_{a}\right)_{b}=\delta_{b a}$. We define matrix elements of the representations of the generators $X^{+}, X^{-}$and $K=q^{H}$ for $\left(\pi_{q}^{p}, V^{(p)}\right)$ as follows.

$$
\begin{aligned}
\left(\pi^{p}\left(X^{+}\right)_{q}\right)_{b}^{a} & =\sqrt{[2 p-a]_{q}[a+1]_{q}} \cdot \delta_{a+1, b} \\
\left(\pi^{p}\left(X^{-}\right)_{q}\right)_{b}^{a} & =\sqrt{[2 p-a+1]_{q}[a]_{q}} \delta_{a-1, b} \\
\left(\pi^{p}(H)_{q}\right)_{b}^{a} & =(2 p-2 a) \cdot \delta_{a, b} .
\end{aligned}
$$

Here $a, b$ are nonnegative integers $(a, b=0,1, \cdots)$, and $p \in \mathbf{C}$. It is easy to see that the operators defined in (3.5) satisfy the defining relations of the algebra $U_{q}(\operatorname{sl}(2))$ with $q$ generic. We call the representation in (3.5) infinite dimensional color representation. We write the basis vector $e_{a}(p)$ by $\mid p, z>_{q}$, and the representation $\left(\pi_{q}^{p}, V^{(p)}\right)$ by $V_{q}^{(p)}$.

Let us discuss decomposition of the tensor product $V_{q}^{p_{1}} \otimes V_{q}^{\left(p_{2}\right)}$. We define the Clebsch-Gordan coefficients by the following

$$
\left|p_{1}, p_{2} ; p_{3}, z_{3}>_{q}=\sum_{z_{1}, z_{2}} C\left(p_{1}, p_{2}, p_{3} ; z_{1}, z_{2}, z_{3}\right)_{q}\right| p_{1}, z_{1}>_{q} \otimes \mid p_{2}, z_{2}>_{q} .
$$

From the comultiplication rule we can show

$$
C\left(p_{1}, p_{2}, p_{3} ; z_{1}, z_{2}, z_{3}\right)_{q}=0 \text {, unless } \quad p_{1}-z_{1}+p_{2}-z_{2}=p_{3}-z_{3} .
$$

The relation (3.7) leads to the charge conservation law. Using (3.7) and the condition $z_{i} \in \mathbf{Z}_{\geq 0}$, we can show

$$
C\left(p_{1}, p_{2}, p_{3} ; z_{1}, z_{2}, z_{3}\right)_{q}=0 \text {, unless } \quad p_{3}=p_{1}+p_{2}-n, n \in \mathbf{Z}_{\geq 0}
$$

Thus we have following.

$$
\mid p_{1}, p_{2} ; p_{1}+p_{2}-n, z_{3}>_{q}
$$




$$
=\sum_{z_{1}=0}^{z_{3}+n} C\left(p_{1}, p_{2}, p_{1}+p_{2}-n ; z_{1}, z_{3}+n-z_{1}, z_{3}\right)_{q}\left|p_{1}, z_{1}>_{q} \otimes\right| p_{2}, z_{3}+n-z_{1}>_{q} .
$$

Here $n \in \mathbf{Z}_{\geq 0}$. The Clebsch-Gordan coefficients for infinite dimensional representations are given by the following.

$$
\begin{aligned}
& C\left(p_{1}, p_{2}, p_{1}+p_{2}-n ; z_{1}, z_{2}, z_{3}\right)_{q}=\delta\left(z_{3}, z_{1}+z_{2}-n\right) \\
& \times \sqrt{\left[2 p_{1}+2 p_{2}-2 n+1\right]_{q}} \sqrt{[n]_{q} !\left[z_{1}\right]_{q} !\left[z_{2}\right]_{q} !\left[z_{3}\right]_{q} !} \\
& \times q^{\left(n-n^{2}\right) / 2+\left(n-z_{2}\right) p_{1}+\left(n+z_{1}\right) p_{2}} \\
& \times \sum_{\nu} \frac{(-1)^{\nu} q^{-\nu\left(2 p_{1}+2 p_{2}-n+1\right)}}{[\nu]_{q} ![n-\nu]_{q} !\left[z_{1}-\nu\right]_{q} !\left[z_{2}-n+\nu\right]_{q} !} \\
& \times \sqrt{\frac{\left[2 p_{1}-n ; z_{1}-\nu\right]_{q} !\left[2 p_{1}-z_{1} ; n-\nu\right]_{q} !\left[2 p_{2}-n ; z_{2}+\nu-n\right]_{q} !\left[2 p_{2}-z_{2} ; \nu\right]_{q} !}{\left[2 p_{1}+2 p_{2}-n+1 ; z_{1}+z_{2}+1\right]_{q} !}} .
\end{aligned}
$$

Here $p_{3}=p_{1}+p_{2}-n, n \in \mathbf{Z}_{\geq 0}$, and the sum over the integer $\nu$ in (3.10) is taken under the following condition

$$
\max \left\{0, n-z_{2}\right\} \leq \nu \leq \min \left\{n, z_{1}\right\}
$$

We shall derive the symmetric expression (3.10) of the Clebsch-Gordan coefficients (3.10) in Appendix A.

Let $V_{q}^{(j)}$ be the spin $j$ representation of $U_{q}(s l(2))$. Let us discuss decomposition of the tensor product $V_{q}^{\left(p_{1}\right)} \otimes V_{q}^{\left(j_{2}\right)}$ and $V_{q}^{\left(j_{1}\right)} \otimes V_{q}^{\left(p_{2}\right)}$. It is easy to see the following rules.

$$
\begin{aligned}
& C\left(\mu_{1}, \mu_{2}, p_{3} ; z_{1}, z_{2}, z_{3}\right)_{q}=0, \\
& \text { unless } \quad \mu_{1}-z_{1}+\mu_{2}-z_{2}=p_{3}-z_{3}, p_{3}=\mu_{1}+\mu_{2}-n \quad\left(n \in \mathbf{Z}_{\geq 0}\right), \\
& \text { and }\left\{\begin{array}{l}
0 \leq n \leq 2 j_{2}, 0 \leq z_{2} \leq 2 j_{2}, \text { if }\left(\mu_{1}, \mu_{2}\right)=\left(p_{1}, j_{2}\right), \\
0 \leq n \leq 2 j_{1}, 0 \leq z_{1} \leq 2 j_{1}, \text { if }\left(\mu_{1}, \mu_{2}\right)=\left(j_{1}, p_{2}\right) .
\end{array}\right.
\end{aligned}
$$


The Clebsch-Gordan coefficients are given by

$$
\begin{aligned}
& C\left(\mu_{1}, \mu_{2}, \mu_{1}+\mu_{2}-n ; z_{1}, z_{2}, z_{3}\right)_{q}=\delta\left(z_{3}, z_{1}+z_{2}-n\right) \\
& \quad \times \sqrt{\left[2 \mu_{1}+2 \mu_{2}-2 n+1\right]_{q}} \sqrt{[n]_{q} !\left[z_{1}\right]_{q} !\left[z_{2}\right]_{q} !\left[z_{3}\right]_{q} !} \\
& \quad \times q^{\left(n-n^{2}\right) / 2+\left(n-z_{2}\right) \mu_{1}+\left(n+z_{1}\right) \mu_{2}} \\
& \quad \times \sum_{\nu} \frac{(-1)^{\nu} q^{-\nu\left(2 \mu_{1}+2 \mu_{2}-n+1\right)}}{[\nu]_{q} ![n-\nu]_{q} !\left[z_{1}-\nu\right]_{q} !\left[z_{2}-n+\nu\right]_{q} !} \\
& \times\left(\frac{\left[2 \mu_{1}-n ; z_{1}-\nu\right]_{q} !\left[2 \mu_{1}-z_{1} ; n-\nu\right]_{q} !\left[2 \mu_{2}-n ; z_{2}+\nu-n\right]_{q} !\left[2 \mu_{2}-z_{2} ; \nu\right]_{q} !}{\left[2 \mu_{1}+2 \mu_{2}-n+1 ; z_{1}+z_{2}+1\right]_{q} !}\right)^{1 / 2} .
\end{aligned}
$$

Here the sum over the integer $\nu$ in (3.13) is taken under the following conditions.

Case (1): $\mu_{1}=p_{1}, \mu_{2}=j_{2}$

$$
\max \left\{0, n-z_{2}\right\} \leq \nu \leq \min \left\{n, z_{1}, 2 j_{2}-z_{2}\right\} .
$$

Case (2): $\mu_{1}=j_{1}, \mu_{2}=p_{2}$

$$
\max \left\{0, n-z_{2}, n+z_{1}-2 j_{1}\right\} \leq \nu \leq \min \left\{n, z_{1}\right\} \text {. }
$$

For convenience we give the Clebsch-Gordan coefficients for finite dimensional representations $V^{\left(j_{i}\right)},(i=1,2,3)$ in the following [23] (see also 24]).

$$
\begin{aligned}
& C\left(j_{1}, j_{2}, j_{3} ; z_{1}, z_{2}, z_{3}\right)_{q}=\delta\left(z_{3}, z_{1}+z_{2}-n\right) \\
& \times\left(\left[2 j_{3}+1\right]_{q}\right)^{1 / 2} \Delta_{q}\left(j_{1} j_{2} j_{3}\right) \\
& \times q^{j_{1}\left(j_{1}+1\right)+j_{2}\left(j_{2}+1\right)-j_{3}\left(j_{3}+1\right)+2\left(j_{1} j_{2}+j_{1}\left(j_{2}-z_{2}\right)-j_{2}\left(j_{1}-z_{1}\right)\right) / 2} \\
& \times\left(\left[2 j_{1}-z_{1}\right]_{q} !\left[z_{1}\right]_{q} !\left[2 j_{2}-z_{2}\right]_{q} !\left[z_{2}\right]_{q} !\left[2 j_{3}-z_{3}\right]_{q} !\left[z_{3}\right]_{q} !\right)^{1 / 2} \\
& \times \sum_{\nu} \frac{(-1)^{\nu}}{[\nu]_{q} !} \frac{q^{-\nu\left(j_{1}+j_{2}+j_{3}+1\right)}}{\left[j_{1}+j_{2}-j_{3}-\nu\right]_{q} !\left[z_{1}-\nu\right]_{q} !} \\
& \times \frac{1}{\left[2 j_{2}-z_{2}-\nu\right]_{q} !\left[j_{3}+j_{1}-j_{2}-z_{1}+\nu\right]_{q} !\left[j_{3}-j_{1}-j_{2}+z_{2}+\nu\right]_{q} !} .
\end{aligned}
$$


Here the sum over the integer $\nu$ is such that

$$
\max \left\{0, j_{2}-j_{3}-j_{1}+z_{1}, j_{1}+j_{2}-j_{3}-z_{2}\right\} \leq \nu \leq \min \left\{z_{1}, j_{1}+j_{2}-j_{3}, 2 j_{2}-z_{2}\right\}
$$

The symbol $\Delta_{q}(a b c)$ has been defined by

$$
\Delta_{q}(a b c)=\left(\frac{[a+b-c]_{q} ![c+a-b]_{q} ![b+c-a]_{q} !}{[a+b+c+1]_{q} !}\right)^{1 / 2} .
$$

If we replace $p_{i}$ by $j_{i}(i=1,2,3)$, then the formal expression of the ClebschGordan coefficients for infinite dimensional representations is consistent with the finite dimensional one (3.16).

The Clebsch-Gordan coefficients satisfy the orthogonality relations for the cases $\left(\mu_{1}, \mu_{2}\right)=\left(p_{1}, p_{2}\right),\left(p_{1}, j_{2}\right)$, and $\left(j_{1}, p_{2}\right)$.

$$
\begin{array}{rl}
\delta_{n n^{\prime}}=\sum_{z_{1}} & C\left(\mu_{1}, \mu_{2}, \mu_{1}+\mu_{2}-n ; z_{1}, \tau-z_{1}, \tau-n\right)_{q} \\
\times & C\left(\mu_{1}, \mu_{2}, \mu_{1}+\mu_{2}-n^{\prime} ; z_{1}, \tau-z_{1}, \tau-n^{\prime}\right)_{q},
\end{array}
$$

We can calculate the Racah coefficients using the Clebsch-Gordan coefficients and the orthogonality relations $(3.19)$ as in $\S 2$. The Racah coefficients are given by

$$
\begin{aligned}
R_{\mu^{\prime \prime} \mu^{\prime}}(q)= & \sqrt{\left[2 \mu^{\prime \prime}+1\right]_{q}\left[2 \mu^{\prime}+1\right]_{q}} W\left(\mu_{1}, \mu_{2}, \mu, \mu_{3} ; \mu^{\prime}, \mu^{\prime \prime}\right)_{q} \\
= & \sum_{w_{1}} \sum_{w_{2}} C\left(\mu_{1}, \mu_{2}, \mu^{\prime} ; w_{1}, w_{2}, w_{1}+w_{2}-n^{\prime}\right)_{q} \\
& \times C\left(\mu^{\prime}, \mu_{3}, \mu ; w_{1}+w_{2}-n^{\prime}, z^{\prime \prime}-w_{2}, w_{1}+z^{\prime \prime}-n\right)_{q} \\
& \times C\left(\mu_{2}, \mu_{3}, \mu_{2}+\mu_{3}-n^{\prime \prime} ; w_{2}, z^{\prime \prime}-w_{2}, z^{\prime \prime}-n^{\prime \prime}\right)_{q} \\
& \times C\left(\mu_{1}, \mu^{\prime \prime}, \mu ; w_{1}, z^{\prime \prime}, w_{1}+z^{\prime \prime}+n^{\prime \prime}-n\right)_{q} .
\end{aligned}
$$

Here we have assumed that $2 \mu_{i}(i=1,2,3)$ is either a nonnegative integer $\left(2 \mu_{i} \in \mathbf{Z}_{\geq 0}\right)$ or a complex parameter $\left(\mu_{i} \in \mathbf{C}\right)$. We have defined $\mu, \mu^{\prime}, \mu^{\prime \prime}$ by

$$
\mu=\mu_{1}+\mu_{2}+\mu_{3}-n, \mu^{\prime}=\mu_{1}+\mu_{2}-n^{\prime}, \mu^{\prime \prime}=\mu_{2}+\mu_{3}-n^{\prime \prime} .
$$


where $n, n^{\prime}, n^{\prime \prime} \in \mathbf{Z}_{\geq 0}$.

\subsection{Finite dimensional cases}

Let $\omega$ be a primitive $N$-th root of unity:

$$
\omega=\exp \left(\frac{2 \pi i s}{N}\right), \quad(N, s)=1
$$

Here the symbol $(a, b)=1$ denotes that the integers $a$ and $b$ have no common divisor except 1 . We write by $\epsilon$ a square root of $\omega: \epsilon=\omega^{1 / 2}=$ $\exp (\pi i s / N), \quad(N, s)=1$.

We take the limit $q \rightarrow \epsilon$ in the infinite dimensional representation (3.5). Then we have

$$
\lim _{q \rightarrow \epsilon}\left(\pi^{p}\left(X^{-}\right)_{q}\right)_{N-1}^{N}=0
$$

From the property 3.23 we can restrict the infinite dimensions into $N$ dimensions: $V_{q}^{(p)}(\infty) \rightarrow V_{\epsilon}^{(p)}(N)$, where $V_{\epsilon}^{(p)}(N)$ is an $N$-dimensional vector space with basis $e_{0}, \cdots, e_{N-1}$. For $a, b=0,1, \cdots N-1$ we have the following matrix representations

$$
\begin{aligned}
\left(\pi^{p}\left(X^{+}\right)_{q=\epsilon}\right)_{b}^{a} & =\left([2 p-a]_{\epsilon}[a+1]_{\epsilon}\right)^{1 / 2} \cdot \delta_{a+1, b} \\
\left(\pi^{p}\left(X^{-}\right)_{q=\epsilon}\right)_{b}^{a} & =\left([2 p-a+1]_{\epsilon}[a]_{\epsilon}\right)^{1 / 2} \cdot \delta_{a-1, b} \\
\left(\pi^{p}(K)_{q=\epsilon}\right)_{a b} & =\epsilon^{(2 p-2 a)} \cdot \delta_{a, b} .
\end{aligned}
$$

We write the finite dimensional color representation $\left\{\pi_{\epsilon}^{p}, V^{(p)}\right\}$ by $V_{\epsilon}^{(p)}$. We write the basis vector $e_{a}$ by $\mid p, z>_{\epsilon}$. The basis vectors for $V_{\epsilon}^{(p)}$ are $\left\{\mid p, z>_{\epsilon}\right.$ $, z=0, \cdots, N-1\}$.

Let us discuss decomposition of the tensor product $V_{\epsilon}^{\left(p_{1}\right)} \otimes V_{\epsilon}^{\left(p_{2}\right)}$. We take the limit $q \rightarrow \epsilon$ in the expression of the Clebsch-Gordan coefficients (3.10). Since there is no singularity in the limiting process in the expression of the 
Clebsch-Gordan coefficients, we have the fusion rule in the following.

$$
V_{\epsilon}^{\left(p_{1}\right)} \otimes V_{\epsilon}^{\left(p_{2}\right)}=\sum_{p_{3}} N_{p_{1}, p_{2}}^{p_{3}} V_{\epsilon}^{\left(p_{3}\right)}
$$

where

$$
\begin{aligned}
N_{p_{1}, p_{2}}^{p_{3}} & =1, \text { for } p_{3}=p_{1}+p_{2}, p_{1}+p_{2}-1, \cdots, p_{1}+p_{2}-N+1 \\
& =0, \text { otherwise. }
\end{aligned}
$$

We note that the condition $0 \leq n \leq N-1$ is derived from the factor $\sqrt{[n]_{q}}$ ! in $(3.10)$. In terms of the Clebsch-Gordan coefficients we have

$$
\begin{aligned}
& C\left(p_{1}, p_{2}, p_{3} ; z_{1}, z_{2}, z_{3}\right)_{\epsilon}=0, \\
& \text { unless } p_{1}-z_{1}+p_{2}-z_{2}=p_{3}-z_{3}, p_{3}=p_{1}+p_{2}-n \text {, } \\
& \quad 0 \leq z_{i} \leq N-1 \text { for } i=1,2,3 \text {, and } 0 \leq n \leq N-1, n \in \mathbf{Z}_{\geq 0} .
\end{aligned}
$$

By taking the limit $q \rightarrow \epsilon$ in (3.10) we obtain the Clebsch-Gordan coefficients for finite dimensional color representations. Let us set $p_{3}=p_{1}+p_{2}-n$, where $n$ is an integer with $0 \leq n \leq N-1$, and $0 \leq z_{i} \leq N-1$, for $i=1,2,3$. The Clebsch-Gordan coefficients for $V_{\epsilon}^{\left(p_{i}\right)}$ for $i=1,2,3$ are given as follows.

$$
\begin{aligned}
& C\left(p_{1}, p_{2}, p_{1}+p_{2}-n ; z_{1}, z_{2}, z_{3}\right)_{\epsilon}=\delta\left(z_{3}, z_{1}+z_{2}-n\right) \\
& \times \sqrt{\left[2 p_{1}+2 p_{2}-2 n+1\right]_{\epsilon}} \sqrt{[n]_{\epsilon} !\left[z_{1}\right]_{\epsilon} !\left[z_{2}\right]_{\epsilon} !\left[z_{3}\right]_{\epsilon} !} \\
& \times \epsilon^{\left(n-n^{2}\right) / 2+\left(n-z_{2}\right) p_{1}+\left(n+z_{1}\right) p_{2}} \\
& \times \sum_{\nu} \frac{(-1)^{\nu} \epsilon^{-\nu\left(p_{1}+p_{2}+p_{3}+1\right)}}{[\nu]_{\epsilon} ![n-\nu]_{\epsilon} !\left[z_{1}-\nu\right]_{\epsilon} !\left[z_{2}-n+\nu\right]_{\epsilon} !} \\
& \times \sqrt{\frac{\left[2 p_{1}-n ; z_{1}-\nu\right]_{\epsilon} !\left[2 p_{1}-z_{1} ; n-\nu\right]_{\epsilon} !\left[2 p_{2}-n ; z_{2}+\nu-n\right]_{\epsilon} !\left[2 p_{2}-z_{2} ; \nu\right]_{\epsilon} !}{\left[2 p_{1}+2 p_{2}-n+1 ; z_{1}+z_{2}+1\right]_{\epsilon} !}} .
\end{aligned}
$$


Here the sum over the integer $\nu$ in (3.28) is taken under the following condition

$$
\max \left\{0, n-z_{2}\right\} \leq \nu \leq \min \left\{n, z_{1}\right\}
$$

Let $V_{\epsilon}^{(j)}$ be the spin $j$ representation of $U_{\epsilon}(s l(2))$. When $q$ is a root of unity $\left(q^{2 N}=1\right)$, the spin $j$ representations of $U_{q}(s l(2))$ has the following constraint. [25, 7]

$$
0 \leq 2 j \leq N-2, \quad 2 j \in \mathbf{Z}_{\geq 0}
$$

Let us discuss decomposition of the tensor product

$$
V_{\epsilon}^{\left(\mu_{1}\right)} \otimes V_{\epsilon}^{\left(\mu_{2}\right)}=\sum_{p_{3}} N_{\mu_{1} \mu_{2}}^{p_{3}} V_{\epsilon}^{\left(p_{3}\right)}
$$

where $\left(\mu_{1}, \mu_{2}\right)=\left(p_{1}, j_{2}\right),\left(j_{1}, p_{2}\right)$. By taking the limit $q \rightarrow \epsilon$ in (3.13) we have the following fusion rule.

Case (1): $\mu_{1}=p_{1}, \mu_{2}=j_{2} \quad\left(2 j_{2}<N-1\right)$

$$
\begin{aligned}
N_{p_{1} j_{2}}^{p_{3}} & =1 \text { for } p_{3}=p_{1}+j_{2}-n, \quad 0 \leq n \leq 2 j_{2}, n \in \mathbf{Z} \\
& =0, \quad \text { otherwise. }
\end{aligned}
$$

Case $(2): \mu_{1}=j_{1}, \mu_{2}=p_{2} \quad\left(2 j_{1}<N-1\right)$

$$
\begin{aligned}
N_{j_{1} p_{2}}^{p_{3}} & =1 \text { for } p_{3}=j_{1}+p_{2}-n, \quad 0 \leq n \leq 2 j_{1}, n \in \mathbf{Z} \\
& =0, \quad \text { otherwise. }
\end{aligned}
$$

By taking the limit: $q \rightarrow \epsilon$ in (3.13), we have the Clebsch-Gordan coefficients. Let us set $p_{3}=\mu_{1}+\mu_{2}-n$. When $\left(\mu_{1}, \mu_{2}\right)=\left(p_{1}, j_{2}\right)$, we assume that $0 \leq z_{i} \leq N-1(i=1,3), 0 \leq z_{2} \leq 2 j_{2}$ and $0 \leq n \leq 2 j_{2}$. When $\left(\mu_{1}, \mu_{2}\right)=\left(j_{1}, p_{2}\right)$, we assume that $0 \leq z_{i} \leq N-1(i=2,3), 0 \leq z_{1} \leq 2 j_{1}$ and $0 \leq n \leq 2 j_{1}$. The Clebsch-Gordan coefficients are given in the following.

$$
C\left(\mu_{1}, \mu_{2}, \mu_{1}+\mu_{2}-n ; z_{1}, z_{2}, z_{3}\right)_{\epsilon}=\delta\left(z_{3}, z_{1}+z_{2}-n\right)
$$




$$
\begin{aligned}
& \times \sqrt{\left[2 \mu_{1}+2 \mu_{2}-2 n+1\right]_{\epsilon}} \sqrt{[n]_{\epsilon} !\left[z_{1}\right]_{\epsilon} !\left[z_{2}\right]_{\epsilon} !\left[z_{3}\right]_{\epsilon} !} \\
& \times \epsilon^{\left(n-n^{2}\right) / 2+\left(n-z_{2}\right) \mu_{1}+\left(n+z_{1}\right) \mu_{2}} \\
& \times \sum_{\nu} \frac{(-1)^{\nu} \epsilon^{-\nu\left(2 \mu_{1}+2 \mu_{2}-n+1\right)}}{[\nu]_{\epsilon} ![n-\nu]_{\epsilon} !\left[z_{1}-\nu\right]_{\epsilon} !\left[z_{2}-n+\nu\right]_{\epsilon} !} \\
& \times\left(\frac{\left[2 \mu_{1}-n ; z_{1}-\nu\right]_{\epsilon} !\left[2 \mu_{1}-z_{1} ; n-\nu\right]_{\epsilon} !\left[2 \mu_{2}-n ; z_{2}+\nu-n\right]_{\epsilon} !\left[2 \mu_{2}-z_{2} ; \nu\right]_{\epsilon} !}{\left[2 \mu_{1}+2 \mu_{2}-n+1 ; z_{1}+z_{2}+1\right]_{\epsilon} !}\right)^{1 / 2} .
\end{aligned}
$$

Here the sum over the integer $\nu$ in (3.34) is taken under the following condition.

Case (1): $\mu_{1}=p_{1}, \mu_{2}=j_{2}$

$$
\max \left\{0, n-z_{2}\right\} \leq \nu \leq \min \left\{n, z_{1}, 2 j_{2}-z_{2}\right\}
$$

Case (2): $\mu_{1}=j_{1}, \mu_{2}=p_{2}$

$$
\max \left\{0, n-z_{2}, n+z_{1}-2 j_{1}\right\} \leq \nu \leq \min \left\{n, z_{1}\right\}
$$

The Clebsch-Gordan coefficients have the following orthogonality relations for the cases $\left(\mu_{1}, \mu_{2}\right)=\left(p_{1}, p_{2}\right),\left(p_{1}, j_{2}\right),\left(j_{1}, p_{2}\right)$.

$$
\begin{aligned}
\delta_{n n^{\prime}}= & \sum_{z_{1}} C\left(\mu_{1}, \mu_{2}, \mu_{1}+\mu_{2}-n ; z_{1}, \tau-z_{1}, \tau-n\right)_{\epsilon} \\
& \times C\left(\mu_{1}, \mu_{2}, \mu_{1}+\mu_{2}-n^{\prime} ; z_{1}, \tau-z_{1}, \tau-n^{\prime}\right)_{\epsilon}, \\
\delta_{z_{1} z_{1}^{\prime}}= & \sum_{n} C\left(\mu_{1}, \mu_{2}, \mu_{1}+\mu_{2}-n ; z_{1}, z+n-z_{1}, z\right)_{\epsilon} \\
& \times C\left(\mu_{1}, \mu_{2}, \mu_{1}+\mu_{2}-n ; z_{1}^{\prime}, z+n-z_{1}^{\prime}, z\right)_{\epsilon} .
\end{aligned}
$$

Recall that $2 \mu_{i}(i=1,2,3)$ be either a nonnegative integer $\left(2 \mu_{i} \in \mathbf{Z}_{\geq 0}\right)$ or a complex parameter $\left(\mu_{i} \in \mathbf{C}\right)$. We introduce $\mu, \mu^{\prime}, \mu^{\prime \prime}$ by

$$
\mu=\mu_{1}+\mu_{2}+\mu_{3}-n, \mu^{\prime}=\mu_{1}+\mu_{2}-n^{\prime}, \mu^{\prime \prime}=\mu_{2}+\mu_{3}-n^{\prime \prime} .
$$


where $n, n^{\prime}, n^{\prime \prime} \in \mathbf{Z}_{\geq 0}$. In terms of the Clebsch-Gordan coefficients the Racah coefficients $W\left(\mu_{1}, \mu_{2}, \mu, \mu_{3} ; \mu^{\prime}, \mu^{\prime \prime}\right)_{\epsilon}$ are given by the following.

$$
\begin{aligned}
R_{\mu^{\prime \prime} \mu^{\prime}}(\epsilon)= & \sqrt{\left[2 \mu^{\prime}+1\right]_{\epsilon}\left[2 \mu^{\prime \prime}+1\right]_{\epsilon}} W\left(\mu_{1}, \mu_{2}, \mu, \mu_{3} ; \mu^{\prime}, \mu^{\prime \prime}\right)_{\epsilon} \\
= & \sum_{w_{1}} \sum_{w_{2}} C\left(\mu_{1}, \mu_{2}, \mu^{\prime} ; w_{1}, w_{2}, w_{1}+w_{2}-n^{\prime}\right)_{\epsilon} \\
& \times C\left(\mu^{\prime}, \mu_{3}, \mu ; w_{1}+w_{2}-n^{\prime}, z^{\prime \prime}-w_{2}, w_{1}+z^{\prime \prime}-n\right)_{\epsilon} \\
& \times C\left(\mu_{2}, \mu_{3}, \mu_{2}+\mu_{3}-n^{\prime \prime} ; w_{2}, z^{\prime \prime}-w_{2}, z^{\prime \prime}-n^{\prime \prime}\right)_{\epsilon} \\
& \times C\left(\mu_{1}, \mu^{\prime \prime}, \mu ; w_{1}, z^{\prime \prime}, w_{1}+z^{\prime \prime}+n^{\prime \prime}-n\right)_{\epsilon} .
\end{aligned}
$$

Finally we give comments. (1) The finite dimensional color representation in this section is equivalent to the $N$ dimensional representation of $U_{q}(\operatorname{sl}(2))$ with $q^{2 N}=1$ in the reference [7]. We have discussed the representation from the view point of the infinite dimensional representation of $U_{q}(s l(2))$ and the limit $q \rightarrow \epsilon$ introduced in the reference [6], and then through the limit we have obtained the formulas for the Clebsch-Gordan coefficients and the Racah coefficients. (2) Fusion rules similar to (3.26) and (3.32) have been given in the reference [26]. The fusion rules are for the $m$ dimensional representations (semi-periodic representations) of $U_{q}(s l(2))$ with $q^{m}=1$, which are different from the finite dimensional color representations (the $N$ dimensional representation with $q^{2 N}=1$ ).

\section{Colored vertex models}

\subsection{Quantum affine algebra $U_{q}(\hat{s l}(2))$}

We express the Boltzmann weights of the colored vertex models in terms of the Clebsch-Gordan coefficients of color representations. Let us consider the $q$-analog of the universal enveloping algebra $U_{q}(\hat{s} l(2, C))$ of the affine 
Kac-Moody algebra $\hat{s} l(2)$. [22] The generators $\left\{X_{i}^{ \pm}, H_{i} ; i=0,1\right\}$ satisfy the following defining relations $(i, j=0,1)$.

$$
\begin{aligned}
{\left[H_{i}, H_{j}\right] } & =0 \\
{\left[H_{i}, X_{i}^{ \pm}\right] } & = \pm 2 X_{i}^{ \pm}, \quad\left[H_{i}, X_{j}^{ \pm}\right]=\mp 2 X_{j}^{ \pm} \quad(i \neq j), \\
{\left[X_{i}^{+}, X_{j}^{-}\right] } & =\delta_{i j} \frac{q^{H_{i}}-q^{-H_{i}}}{q-q^{-1}} \\
\sum_{\nu=0}^{3}(-1)^{\nu} & \frac{[3]_{q} !}{[3-\nu]_{q} ![\nu]_{q} !}\left(X^{ \pm}\right)^{3-\nu} X_{j}^{ \pm}\left(X_{i}^{ \pm}\right)^{\nu}=0 \quad(i \neq j) .
\end{aligned}
$$

The comultiplication is given by

$$
\begin{aligned}
\Delta\left(H_{i}\right) & =H_{i} \otimes I+I \otimes H_{i}, \\
\Delta\left(X_{i}^{ \pm}\right) & =X_{i}^{ \pm} \otimes q^{H_{i} / 2}+q^{-H_{i} / 2} \otimes X_{i}^{ \pm} .
\end{aligned}
$$

We denote by $\mathcal{R}$ the universal $R$ matrix of $U_{q}(\hat{s} l(2, C))$. The universal $R$ matrix satisfie the following

$$
\mathcal{R} \Delta(a)=\tau \circ \Delta(a) \mathcal{R}, \quad a \in U_{q}(\hat{s} l(2, C)),
$$

where $\tau$ is the permutation operator $\tau\left(t_{1} \otimes t_{2}\right)=t_{2} \otimes t_{1}$, for $t_{1}, t_{2} \in U_{q}(\hat{s} l(2, C))$. For simplicity we sometimes write $U_{q}(\hat{s} l(2, \mathbf{C}))$ and $U_{q}(s l(2, \mathbf{C}))$ by $\hat{U}_{q}$ and $U_{q}$, respectively.

Let us discuss solvable models from the viewpoint of $\hat{U}_{q}$. We define a homomorphism $\phi: \hat{U}_{q} \rightarrow U_{q}$ by $\phi\left(X_{0}^{ \pm}\right)=X^{\mp}, \phi\left(X_{1}^{ \pm}\right)=X^{ \pm}$, and $\phi\left(H_{0}\right)=-H$ , $\phi\left(H_{1}\right)=H$. We introduce an automorphism $T_{x}: \hat{U}_{q} \rightarrow \hat{U}_{q}$ by $T_{x}\left(X_{0}^{ \pm}\right)=$ $x^{ \pm 1} X_{0}^{ \pm}, T_{x}(X)=X$, for $X=X_{1}^{ \pm}, H_{0}, H_{1}$.

We define an operator $R(x)$ by $R(x)=\phi\left(\left(T_{x} \otimes I(\mathcal{R})\right)\right.$. Then the operator $R(x)$ satisfies the following for $i=0,1$.

$$
R(x) q^{\hat{H}_{i} / 2} \otimes q^{\hat{H}_{i} / 2}=q^{\hat{H}_{i} / 2} \otimes q^{\hat{H}_{i} / 2} R(x),
$$




$$
\begin{aligned}
& R(x)\left(x^{\delta(i, 0)} \hat{X}_{i}^{+} \otimes q^{\hat{H}_{i} / 2}+q^{-\hat{H}_{i} / 2} \otimes \hat{X}_{i}^{+}\right) \\
= & \left(x^{\delta(i, 0)} \hat{X}_{i}^{+} \otimes q^{-\hat{H}_{i} / 2}+q^{\hat{H}_{i} / 2} \otimes \hat{X}_{i}^{+}\right) R(x), \\
& R(x)\left(x^{-\delta(i, 0)} \hat{X}_{i}^{-} \otimes q^{\hat{H}_{i} / 2}+q^{-\hat{H}_{i} / 2} \otimes \hat{X}_{i}^{-}\right) \\
= & \left(x^{-\delta(i, 0)} \hat{X}_{i}^{-} \otimes q^{-\hat{H}_{i} / 2}+q^{\hat{H}_{i} / 2} \otimes \hat{X}_{i}^{-}\right) R(x) .
\end{aligned}
$$

Here $\hat{X}_{i}^{ \pm}=\phi\left(X_{i}^{ \pm}\right), \hat{H}_{i}=\phi\left(H_{i}\right)$ for $i=0,1$.

Let us take arbitrary two representations $\mu_{1}, \mu_{2}$ of $U_{q}$. We can take as $\mu_{1}$ and $\mu_{2}$ the spin $j$ representations of $U_{q}(s l(2))$, or the (infinite dim. or finite dim. ) color representations of $U_{q}(s l(2))$. We define $R$ matrix and its matrix elements for the representations by

$$
\begin{aligned}
R_{\mu_{1} \mu_{2}}(x) & =\pi^{\mu_{1}} \otimes \pi^{\mu_{2}}(R(x)), \\
R_{\mu_{1} \mu_{2}}(x)_{b_{1} b_{2}}^{a_{1} a_{2}} & =\left(\pi^{\mu_{1}} \otimes \pi^{\mu_{2}}(R(x))\right)_{b_{1} b_{2}}^{a_{1} a_{2}} .
\end{aligned}
$$

Then the relations (4.4), (4.5) and (4.6) give linear equations for the $R$ matrix elements. It is easy to see that if the matrix elements satisfy the linear equations, then the matrix elements satisfy the Yang-Baxter equations. 27

$$
\begin{aligned}
& \sum_{c_{1} c_{2} c_{3}} R_{p_{1} p_{2}}(u)_{b_{1} b_{2}}^{c_{1} c_{2}} R_{p_{1} p_{3}}(u+v)_{c_{1} b_{3}}^{a_{1} c_{3}} R_{p_{2} p_{3}}(v)_{c_{2} c_{3}}^{a_{2} a_{3}} \\
= & \sum_{c_{1} c_{2} c_{3}} R_{p_{1} p_{2}}(u)_{c_{1} c_{2}}^{a_{1} a_{2}} R_{p_{1} p_{3}}(u+v)_{b_{1} c_{3}}^{c_{1} a_{3}} R_{p_{2} p_{3}}(v)_{b_{2} b_{3}}^{c_{2} c_{3}} .
\end{aligned}
$$

Here we have defined the spectral parameter $u$ by $x=\exp u$. In operator formalism, solutions of the linear equations ( $R$ matrix) can be written as

$$
R(q ; u)=\sum_{\mu}\left|\mu_{1} \mu_{2} ; \mu>g\left(\mu_{1}, \mu_{2}, \mu ; u\right)<\mu_{1} \mu_{2} ; \mu\right|
$$

Here the sum is taken over all $\mu$ appearing in the decomposition of the tensor product $\mu_{1} \otimes \mu_{2}\left(\mu \subset \mu_{1} \otimes \mu_{2}\right)$, and $g\left(\mu_{1}, \mu_{2}, \mu ; u\right)$ is some function of the spectral parameter $x=\exp u$. 


\subsection{Infinite dimensional case}

Let us introduce the colored vetex models. [3, 6] The Boltzmann weights of the colored vertex model $X_{\alpha \beta}(u)_{c d}^{a b}$ is defined for the configuration $\{a, b, c, d\}$. (Fig. 1) The Yang-Baxter relation for the colored vertex model reads

$$
\begin{aligned}
& \sum_{c_{1}, c_{2}, c_{3}} X_{\alpha_{1}, \alpha_{2}}\left(u_{1}-u_{2}\right)_{c_{2} c_{1}}^{a_{1} a_{2}} X_{\alpha_{1}, \alpha_{3}}\left(u_{1}-u_{3}\right)_{c_{3} b_{1}}^{c_{1} a_{3}} X_{\alpha_{2}, \alpha_{3}}\left(u_{2}-u_{3}\right)_{b_{3} b_{2}}^{c_{2} c_{3}} \\
= & \sum_{c_{1}, c_{2}, c_{3}} X_{\alpha_{2}, \alpha_{3}}\left(u_{2}-u_{3}\right)_{c_{3} c_{2}}^{a_{2} a_{3}} X_{\alpha_{1}, \alpha_{3}}\left(u_{1}-u_{3}\right)_{b_{3} c 1}^{a_{1} c_{3}} X_{\alpha_{1}, \alpha_{2}}\left(u_{1}-u_{2}\right)_{b_{2} b 1}^{c_{1} c_{2}} .
\end{aligned}
$$

We construct the colored vertex models by calculating the matrix elements of the $R$ matrix (4.9) on the color representations. Let us consider color

representations $\left(\pi_{q}^{\left(p_{i}\right)}, V^{(p)}\right) \quad(i=1,2)$ of $U_{q}(s l(2))$. We define $R_{p_{1} p_{2}}(q ; u)$ and its matrix elements by

$$
\begin{aligned}
R_{p_{1} p_{2}}(q ; u) & =\pi_{q}^{\left(p_{1}\right)} \otimes \pi_{q}^{\left(p_{2}\right)}(R(x)) \\
R_{p_{1} p_{2}}(q ; u)_{b_{1} b_{2}}^{a_{1} a_{2}} & =\left(\pi_{q}^{\left(p_{1}\right)} \otimes \pi_{q}^{\left(p_{2}\right)}(R(x))\right)_{b_{1} b_{2}}^{a_{1} a_{2}}
\end{aligned}
$$

Here $a_{1}, a_{2}, b_{1}, b_{2}=0,1, \cdots, \infty$.

We can show that the follwoing gives a solution of the linear equations (4.4), (4.5) and (4.6).

$$
\begin{aligned}
R_{p_{1} p_{2}}(q ; u)_{b_{1} b_{2}}^{a_{1} a_{2}}= & \sum_{n}^{\infty} g\left(p_{1}, p_{2}, n ; u\right)_{q} \\
& C\left(p_{1}, p_{2}, p_{1}+p_{2}-n ; b_{1}, b_{2}, b_{1}+b_{2}-n\right)_{q} \\
& C\left(p_{2}, p_{1}, p_{1}+p_{2}-n ; a_{2}, a_{1}, a_{1}+a_{2}-n\right)_{q} .
\end{aligned}
$$

where

$$
g\left(p_{1}, p_{2}, n ; u\right)_{q}=(-1)^{n} \prod_{k=0}^{n-1} \frac{\left[u-p_{1}-p_{2}+k\right]_{q}}{\left[u+p_{1}+p_{2}-k\right]_{q}}, \text { for } n \in \mathbf{Z}_{\geq 0}
$$


We assume $g\left(p_{1}, p_{2}, n=0 ; u\right)_{q}=1$. For the fixed values of $a_{i}, b_{i}(i=1,2)$, the sum in (4.12) reduces to a finite sum. Therefore the sum in (4.12) is well defined. Thus we have an explicit formula for the $R$ matrix elements for the infinite dimensional color representation. It is remarked that the case $p_{1}=p_{2}$, the infinite dimensional representation of the $R$ matrix with a spectral parameter were discussed by Jimbo by using projection operators. 22

Through the $R$ matrix (4.12) the Boltzmann weights of the colored vertex model are written as follows

$$
X_{\alpha \beta}^{(\infty)}(u)_{c d}^{a b}=R_{p_{1} p_{2}}(q ; u)_{c d}^{b a}
$$

where $\alpha=q^{p_{2}}$ and $\beta=q^{p_{1}}$. Thus we obtain the colored vertex model of the infinite state case introduced in the reference [6].

Using the Clebsch-Gordan coeffcients we can calculate $R$ matrix for the representations $V^{j}$ and $V^{(\infty)}$. We define $R_{p_{1} j_{2}}(x)$ and its matrix elements by

$$
\begin{aligned}
R_{p_{1} j_{2}}(x) & =\pi_{q}^{\left(p_{1}\right)} \otimes \pi_{q}^{\left(j_{2}\right)}(R(x)) \\
R_{p_{1} j_{2}}(x)_{b_{1} b_{2}}^{a_{1} a_{2}} & =\left(\pi_{q}^{\left(p_{1}\right)} \otimes \pi_{q}^{\left(j_{2}\right)}(R(x))\right)_{c d}^{a b}
\end{aligned}
$$

Here $a_{1}, b_{1}=0,1, \cdots, N-1$, and $a_{2}, b_{2}=0,1, \cdots 2 j_{2}$. The matrix elements are given by the following.

$$
\begin{aligned}
R_{p_{1} j_{2}}(x)_{b_{1} b_{2}}^{a_{1} a_{2}}= & \sum_{n=0}^{2 j_{2}} g\left(p_{1}, j_{2}, n ; u\right)_{q} \\
& C\left(p_{1}, j_{2}, p_{1}+p_{2}-n ; b_{1}, b_{2}, b_{1}+b_{2}-n\right)_{q} \\
& C\left(j_{2}, p_{1}, p_{1}+p_{2}-n ; a_{2}, a_{1}, a_{1}+a_{2}-n\right)_{q} .
\end{aligned}
$$

Here $g\left(p_{1}, j_{2}, n ; u\right)_{q}$ is given by (4.13) with $p_{2}=j_{2}$. 


\subsection{Finite dimensional case}

Let us consider color representations $\left(\pi_{\epsilon}^{\left(p_{i}\right)}, V^{(N)}\right) \quad(i=1,2)$ of $U_{q}(\operatorname{sl}(2))$ with $q=\epsilon$. We define $R_{p_{1} p_{2}}(\epsilon ; x)$ and its matrix elements by

$$
\begin{aligned}
R_{p_{1} p_{2}}(\epsilon ; x) & =\pi_{\epsilon}^{\left(p_{1}\right)} \otimes \pi_{\epsilon}^{\left(p_{2}\right)}(R(x)), \\
R_{p_{1} p_{2}}(\epsilon ; x)_{b_{1} b_{2}}^{a_{1} a_{2}} & =\left(\pi_{\epsilon}^{\left(p_{1}\right)} \otimes \pi_{\epsilon}^{\left(p_{2}\right)}(R(x))\right)_{b_{1} b_{2}}^{a_{1} a_{2}}
\end{aligned}
$$

Then we have the following.

$$
\begin{aligned}
R_{p_{1} p_{2}}(\epsilon ; u)_{b_{1} b_{2}}^{a_{1} a_{2}}= & \sum_{n=0}^{N-1} g\left(p_{1}, p_{2}, n ; u\right)_{\epsilon} \\
& \times C\left(p_{1}, p_{2}, p_{1}+p_{2}-n ; b_{1}, b_{2}, b_{1}+b_{2}-n\right)_{\epsilon} \\
& \quad \times C\left(p_{2}, p_{1}, p_{1}+p_{2}-n ; a_{2}, a_{1}, a_{1}+a_{2}-n\right)_{\epsilon}
\end{aligned}
$$

where

$$
g\left(p_{1}, p_{2}, n ; u\right)_{\epsilon}=(-1)^{n} \prod_{k=0}^{n-1} \frac{\left[u-p_{1}-p_{2}+k\right]_{\epsilon}}{\left[u+p_{1}+p_{2}-k\right]_{\epsilon}}, \text { for } n \in \mathbf{Z}_{\geq 0}
$$

We assume $g\left(p_{1}, p_{2}, n=0 ; u\right)_{\epsilon}=1$.

Through the $R$ matrix (4.12) the Boltzmann weights weights of the colored vertex model are written as follows

$$
X_{\alpha \beta}^{(N)}(u)_{c d}^{a b}=R_{p_{1} p_{2}}(\epsilon ; u)_{c d}^{b a}
$$

where $\alpha=\epsilon^{p_{2}}$ and $\beta=\epsilon^{p_{1}}$. Thus we obtain the colored vertex model of the finite state case given in the reference [3].

Using the Clebsch-Gordan coeffcients we can calculate $R$ matrix for the representations $V^{j}$ and $V^{(N)}$. We define $R_{p_{1} j_{2}}(x)$ and its matrix elements by

$$
\begin{aligned}
R_{p_{1} j_{2}}(\epsilon ; x) & =\pi_{\epsilon}^{\left(p_{1}\right)} \otimes \pi_{\epsilon}^{\left(j_{2}\right)}(R(x)) \\
R_{p_{1} j_{2}}(\epsilon ; x)_{b_{1} b_{2}}^{a_{1} a_{2}} & =\left(\pi_{q}^{\left(p_{1}\right)} \otimes \pi_{\epsilon}^{\left(j_{2}\right)}(R(x))\right)_{c d}^{a b}
\end{aligned}
$$


Here $a_{1}, b_{1}=0,1, \cdots, N-1$, and $a_{2}, b_{2}=0,1, \cdots 2 j_{2}$. The matrix elements are given by the following.

$$
\begin{aligned}
R_{p_{1} j_{2}}(\epsilon ; x)_{b_{1} b_{2}}^{a_{1} a_{2}}= & \sum_{n}^{2 j_{2}} g\left(p_{1}, j_{2}, n ; u\right)_{\epsilon} \\
& C\left(p_{1}, j_{2}, p_{1}+p_{2}-n ; b_{1}, b_{2}, b_{1}+b_{2}-n\right)_{\epsilon} \\
& C\left(j_{2}, p_{1}, p_{1}+p_{2}-n ; a_{2}, a_{1}, a_{1}+a_{2}-n\right)_{\epsilon} .
\end{aligned}
$$

Here the function $g\left(p_{1}, j_{2}, n ; u\right)_{\epsilon}$ is given by (4.19) with $p_{2}=j_{2}$. It is easy to show that the $R$ matrix (4.22) gives a solution of the linear equation (4.5).

\section{Colored IRF models}

\subsection{Infinite dimensional case}

Using the Racah coefficients for color representations we define colored IRF (Interaction Round a Face) models. Let the symbol $w\left(a, b, c, d ; p_{1}, p_{2} ; u\right)_{q}$ denotes the Boltzmann weight of colored IRF model for the configuration $\left\{a, b, c, d ; p_{1}, p_{2}\right\}$. (Fig. 2) The admissible condition is determined by the fusion rules. In the configuration $\left\{a, b, c, d ; p_{1}, p_{2}\right\}, a$ is admissble to $d$ if $a$ $\subset p_{1} \otimes d$, i.e., $N_{p_{1}, d}^{a} \neq 0$. We denote this condition by $a \sim d$. The Boltzmann weight $w\left(a, b, c, d ; p_{1}, p_{2} ; u\right)_{q}$ is defined to be zero, unless $a \sim d, b \sim a, c \sim d$ and $b \sim c$.

The Yang-Baxter relation for the colored IRF model is given by the following.

$$
\begin{aligned}
& \sum_{g} w\left(g_{1}, g_{2}, g, g_{0} ; p_{1}, p_{2} ; u\right) w\left(g_{2}, g_{3}, g_{2}^{\prime}, g ; p_{1}, p_{3} ; u+v\right) \\
& w\left(g, g_{2}^{\prime}, g_{1}^{\prime}, g_{0} ; p_{2}, p_{3} ; v\right) \\
= & \sum_{g} w\left(g_{2}, g_{3}, g, g_{1} ; p_{2}, p_{3} ; v\right) w\left(g_{1}, g, g_{1}^{\prime}, g_{0} ; p_{1}, p_{3} ; u+v\right)
\end{aligned}
$$




$$
w\left(g, g_{3}, g_{2}^{\prime}, g_{1}^{\prime} ; p_{1}, p_{2} ; u\right)
$$

The Boltzmann weights of the colored IRF models are given as follows.

$$
\begin{aligned}
& w\left(g_{12}, g_{1}, g_{21}, g_{0} ; p_{1}, p_{2} ; u\right)_{q} \\
= & \sum_{n=0}^{\infty} g\left(p_{1}, p_{2}, n ; u\right)_{q}[2 p+1]_{q} \sqrt{\left[2 g_{12}+1\right]_{q}\left[2 g_{21}+1\right]_{q}} \\
& W\left(p_{2}, p_{1}, g_{1}, g_{0} ; p, g_{12}\right)_{q} W\left(p_{2}, g_{1}, p_{1}, g_{0} ; g_{21}, p\right)_{q},
\end{aligned}
$$

where $p=p_{1}+p_{2}-n, n \in \mathbf{Z}_{\geq 0}$, and the function $g\left(p_{1}, p_{2}, n ; u\right)_{q}$ is given by (4.13). We recall that the symbols $W$ and $w$ denote the Racah coefficient and the Boltzmann weight of the IRF model, respectively.

The expression (5.2) can be derived from (4.12) by using the definition of the Racah coefficients [28] (see also [29] for the cases of the spin $j$ representations). Therefore the Boltzmann weights given in (5.2) satisfy the Yang-Baxter relation for the IRF models (5.1).

We can discuss hybrid type colored IRF models, i.e., $2 \mu_{1} \in \mathbf{Z}_{\geq 0}$ or $2 \mu_{2} \in$ $\mathbf{Z}_{\geq 0}$. For simplicity we consider only the case $p_{1} \in \mathbf{C}$ and $2 \mu_{2}=2 j_{2} \in \mathbf{Z}_{\geq 0}$. The Boltzmann weights of the colored IRF models are given as follows.

$$
\begin{aligned}
& w\left(g_{12}, g_{1}, g_{21}, g_{0} ; p_{1}, j_{2} ; u\right)_{q} \\
= & \sum_{n=0}^{2 j_{2}} g\left(p_{1}, j_{2}, n ; u\right)_{q}[2 p+1]_{q} \sqrt{\left[2 g_{12}+1\right]_{q}\left[2 g_{21}+1\right]_{q}} \\
& W\left(j_{2}, p_{1}, g_{1}, g_{0} ; p, g_{12}\right)_{q} W\left(p_{1}, j_{2}, g_{1}, g_{0} ; g_{21}, p\right)_{q},
\end{aligned}
$$

where $p=p_{1}+j_{2}-n, 0 \leq n \leq 2 j_{2}, n \in \mathbf{Z}_{\geq 0}$, and the function $g\left(p_{1}, p_{2}, n ; u\right)_{q}$ is given by (4.13).

\subsection{Finite dimensional case}

Using the Racah coefficients for color representations we define colored IRF (Interaction Round a Face) models. Let the symbol $w\left(a, b, c, d ; p_{1}, p_{2} ; u\right)_{\epsilon}$ 
denote the Boltzmann weight of colored IRF model associated with finite dimensional color representations of $U_{q}(s l(2))$.

The Boltzmann weights of the colored IRF model are given by the following.

$$
\begin{aligned}
& w\left(g_{12}, g_{1}, g_{21}, g_{0} ; p_{1}, p_{2} ; u\right)_{\epsilon} \\
= & \sum_{n=0}^{N-1} g\left(p_{1}, p_{2}, n ; u\right)_{\epsilon}[2 p+1]_{q} \sqrt{\left[2 g_{12}+1\right]_{\epsilon}\left[2 g_{21}+1\right]_{\epsilon}} \\
& \times W\left(p_{2}, p_{1}, g_{1}, g_{0} ; p, g_{12}\right)_{\epsilon} W\left(p_{1}, p_{2}, g_{1}, g_{0} ; p, g_{21}\right)_{\epsilon}
\end{aligned}
$$

Here the function $g\left(p_{1}, p_{2}, n ; u\right)_{\epsilon}$ is given by (4.19).

We can discuss hybrid type colored IRF models, i.e., $2 \mu_{1} \in \mathbf{Z}_{\geq 0}$ or $2 \mu_{2} \in$ $\mathbf{Z}_{\geq 0}$. For simplicity we consider only the case $p_{1} \in \mathbf{C}$ and $2 \mu_{2}=2 j_{2} \in \mathbf{Z}_{\geq 0}$. The Boltzmann weights of the colored IRF models are given as follows.

$$
\begin{aligned}
& w\left(g_{12}, g_{1}, g_{21}, g_{0} ; p_{1}, j_{2} ; u\right)_{\epsilon} \\
= & \sum_{n=0}^{2 j_{2}} g\left(p_{1}, j_{2}, n ; u\right)_{\epsilon}[2 p+1]_{\epsilon} \sqrt{\left[2 g_{12}+1\right]_{\epsilon}\left[2 g_{21}+1\right]_{\epsilon}} \\
& W\left(j_{2}, p_{1}, g_{1}, g_{0} ; p, g_{12}\right)_{\epsilon} W\left(p_{1}, j_{2}, g_{1}, g_{0} ; p, g_{21}\right)_{\epsilon}
\end{aligned}
$$

where $p=p_{1}+j_{2}-n, 0 \leq n \leq 2 j_{2}, n \in \mathbf{Z}_{\geq 0}$, and the function $g\left(p_{1}, j_{2}, n\right)_{\epsilon}$ is given by (4.19).

\section{$6 \quad$ Invariants of trivalent colored oriented graphs}

\subsection{Basic relations for the colored braid matrices}

We construct new invariants of colored oriented graphs using the ClebschGordan coefficients for finite dimensional color representations given in $\S 3$.

Let us define the $N$-state colored braid matrix. We assume the charge conservation: $G_{c d}^{a b}\left(p_{\alpha}, p_{\beta} ; \pm\right)=0$ unless $a+b=c+d\left(\alpha=q^{-4 p_{\alpha}}, \beta=q^{-4 p_{\beta}}\right)$. 
Furthermore we assume $G_{c d}^{a b}\left(p_{\alpha}, p_{\beta} ;+\right)=0$ if $a<d$, and $G_{c d}^{a b}\left(p_{\alpha}, p_{\beta} ;-\right)=0$ if $a>d$. We define $(z ; q)_{n}$ by $(z ; q)_{n}=(1-z)(1-z q) \cdots\left(1-z q^{n-1}\right)$ for $n>0$ and $(z ; q)_{0}=1$ for $n=0$. The nonzero elements are written as follows.

$$
\begin{aligned}
& G_{c, d}^{a, b}\left(p_{\alpha}, p_{\beta} ;+\right)=\epsilon^{-2 b p_{\alpha}-2 d p_{\beta}} \epsilon^{2 b d} \epsilon^{2 p_{\alpha} p_{\beta}} \\
& \left(\frac{\left(\epsilon^{2} ; \epsilon^{2}\right)_{c}}{\left(\epsilon^{2} ; \epsilon^{2}\right)_{b}\left(\epsilon^{2} ; \epsilon^{2}\right)_{c-b}} \frac{\left(\epsilon^{2} ; \epsilon^{2}\right)_{a}}{\left(\epsilon^{2} ; \epsilon^{2}\right)_{d}\left(\epsilon^{2} ; \epsilon^{2}\right)_{a-d}} \frac{\left(\epsilon^{-4 p_{\alpha}} ; \epsilon^{2}\right)_{a}}{\left(\epsilon^{-4 p_{\alpha}} ; \epsilon^{-2}\right)_{d}} \frac{\left(\epsilon^{-4 p_{\beta}} ; \epsilon^{2}\right)_{c}}{\left(\epsilon^{-4 p_{\beta}} ; \epsilon^{2}\right)_{b}}\right)^{1 / 2}, \\
& G_{c, d}^{a, b}\left(p_{\alpha}, p_{\beta} ;-\right)=\epsilon^{2 a p_{\alpha}+2 c p_{\beta}} \epsilon^{-2 a c} \epsilon^{-2 p_{\alpha} p_{\beta}} \\
& \left(\frac{\left(\epsilon^{-2} ; \epsilon^{-2}\right)_{b}}{\left(\epsilon^{-2} ; \epsilon^{-2}\right)_{b-c}\left(\epsilon^{-2} ; \epsilon^{-2}\right)_{c}} \frac{\left(\epsilon^{-2} ; \epsilon^{-2}\right)_{d}}{\left(\epsilon^{-2} ; \epsilon^{-2}\right)_{d-a}\left(\epsilon^{-2} ; \epsilon^{-2}\right)_{a}} \frac{\left(\epsilon^{4 p_{\alpha}} ; \epsilon^{-2}\right)_{b}}{\left(\epsilon^{4 p_{\alpha}} ; \epsilon^{-2}\right)_{c}} \frac{\left(\epsilon^{4 p_{\beta}} ; \epsilon^{-2}\right)_{d}}{\left(\epsilon^{4 p_{\beta}} ; \epsilon^{-2}\right)_{a}}\right)^{1 / 2} .
\end{aligned}
$$

Then the colored braid matrix satisfies the following relation.

$$
\begin{aligned}
& \sum_{c_{1}, c_{2}, c_{3}} G_{c_{2}, c_{1}}^{a_{1}, a_{2}}\left(p_{1}, p_{2} ;+\right) G_{c_{3}, b_{1}}^{c_{1}, a_{3}}\left(p_{1}, p_{3} ;+\right) G_{b_{3}, b_{2}}^{c_{2}, c_{3}}\left(p_{2}, p_{3} ;+\right) \\
= & \sum_{c_{1}, c_{2}, c_{3}} G_{c_{3}, c_{2}}^{a_{2}, a_{3}}\left(p_{2}, p_{3} ;+\right) G_{b_{3}, c_{1}}^{a_{1}, c_{3}}\left(p_{1}, p_{3} ;+\right) G_{b_{2}, b_{1}}^{c_{1}, c_{2}}\left(p_{1}, p_{2} ;+\right) .
\end{aligned}
$$

Here $p_{1}, p_{2}, p_{3}$ denote the colors of the strings.

It has been explicitly shown that the colored braid matrix is equivalent to the $R$ matrix of the color representations of $U_{q}(s l(2))$. [6]

Let us introduce basic relations for the colored braid matrix and the Clebsch-Gordan coefficients. We note they are related to the Reidemeister moves.

(i) First inversion relation:

$$
\sum_{e, f} G_{e f}^{a b}\left(p_{1}, p_{2} ;+\right) G_{c d}^{e f}\left(p_{1}, p_{2} ;-\right)=\sum_{e, f} G_{e f}^{a b}\left(p_{1}, p_{2} ;-\right) G_{c d}^{e f}\left(p_{1}, p_{2} ;+\right)=\delta_{c}^{a} \delta_{d}^{b} .
$$

(ii) Second inversion relation:

$$
\sum_{e, f} G_{c f}^{a e}\left(p_{1}, p_{2} ;+\right) G_{b e}^{d f}\left(p_{1}, p_{2} ;-\right) \omega^{d-e}=\delta_{b}^{a} \delta_{d}^{c}
$$




$$
\sum_{e, f} G_{c f}^{a e}\left(p_{2}, p_{1} ;-\right) G_{b e}^{d f}\left(p_{2}, p_{1} ;+\right) \omega^{d-e}=\delta_{b}^{a} \delta_{d}^{c}
$$

(iii) Markov trace property:

$$
\sum_{b} G_{a b}^{a b}(p, p ; \pm) \omega^{-b}=\epsilon^{2 p(N-1) \pm 2 p(N-1) \pm 2 p^{2}} .
$$

(iv)

$$
\begin{aligned}
& \sum_{c_{1} c_{2}} G_{b_{3} c_{1}}^{a_{1} c_{3}}\left(p_{1}, p_{3} ;+\right) G_{c_{3} c_{2}}^{a_{2} a_{3}}\left(p_{2}, p_{3} ;+\right) C\left(p_{1}, p_{2}, p ; c_{1}, c_{2}, b\right)_{\epsilon} \\
= & \sum_{c} C\left(p_{1}, p_{2}, p ; a_{1}, a_{2}, c\right)_{\epsilon} G_{b_{3} b}^{c a_{3}}\left(p, p_{3} ;+\right), \\
& \sum_{c_{1} c_{2}} G_{b_{3} c_{1}}^{a_{1} c_{3}}\left(p_{3}, p_{1} ;-\right) G_{c_{3} c_{2}}^{a_{2} a_{3}}\left(p_{3}, p_{2} ;-\right) C\left(p_{1}, p_{2}, p ; c_{1}, c_{2}, b\right)_{\epsilon} \\
= & \sum_{c} C\left(p_{1}, p_{2}, p ; a_{1}, a_{2}, c\right)_{\epsilon} G_{b_{3} b}^{c a_{3}}\left(p_{3}, p ;-\right) .
\end{aligned}
$$

\subsection{Invariants of colored oriented framed tangle graphs with trivalent vertices}

We consider trivalent graphs, which have vertices with three edges. Framed graphs have framing vector fields. We assume that all three edges have common tangent vector in the trivalent vertex, and the framing vector field is always normal to the tangent vector to the vertex. (Fig. 3)

We introduce "tangle graph". We define $(k, l)$-oriented tangle graph $T$ by a finite set of disjoint oriented arcs, oriented trivalent vertices, and oriented circles properly embedded in $\mathbf{R}^{2} \times[0,1]$ such that

$$
\partial T=\{(i, 0,0) ; i=1,2, \cdots, k\} \cup\{(j, 0,1) ; j=1, \cdots, l\},
$$

where $\partial T$ denotes the upper and lower boundaries of the tangle graph. (Fig. 4) We define colored oriented tangle graphs $(T, \boldsymbol{\alpha})$ by assigning colors on edges, arcs and circles of tangle graphs. 
We can express trivalent framed tangle graph by plane diagram. [30, 31, 32. We can choose the diagram such that the framing vector field is normal to the plane of projection and directed "up".

Proposition 6.1 30 The isotopy invariants of framed tangle graphs are functions on their plane diagrams, invariant under the following local moves $D_{1} \sim D_{7}$.

(Fig. 5)

We introduce weights for diagrams. We assign the colored braid matrices, the Clebsch-Gordan coefficients, and the weights $U_{i}$ to the braiding diagrams, trivalent vertex diagrams, and the creation-annihilation diagrams, respectively. (Fig. 6)

We define $\phi(T, \boldsymbol{\alpha})$ for a tangle graph $T$ by the summation over all possible configurations of variables $z_{i}$ on the edges (or segments) of the graph. In the summation we fix the colors $p_{i}$. The sum corresponds to partition function in statistical mechanics. Then from the relations (6.2), (6.3), (6.4), (6.5) the sum $\phi(T, \boldsymbol{\alpha})$ for the tangle graph $T$ is invariant under the moves $D_{1} \sim D_{7}$. Thus we have isotopy invariants of trivalent colored oriented framed tangle graphs.

\subsection{Invariants of trivalent framed graphs}

We construct invariants of trivalent colored oriented framed graphs by an approach parallel to that for the colored link invariants. [5] Through $(1,1)$ tangle graph we introduce another invariant of a framed graph. [5]

Let $T$ be a $(1,1)$ - tangle graph. We denote by $\hat{T}$ the graph obtained by closing the open strings of $T$. It is easy to show the following proposition. [5] 
Proposition 6.2 Let $T_{1}$ and $T_{2}$ denote two $(1,1)$-tangle graph. If $\hat{T}_{1}$ is isotopic to $\hat{T}_{2}$ as a graph in $S^{3}$ by an isotopy which carries the closing component of $\hat{T}_{1}$ to that of $\hat{T}_{2}$. Then $T_{1}$ is isotopic to $T_{2}$ as a $(1,1)$-tangle graph.

Let $T$ be a $(1,1)$-tangle graph. We put $F=\hat{T}$ and $s$ is the color of the closing component (or edge) of $\hat{T}$. We denote by $\phi(T, \boldsymbol{\alpha})_{b}^{a}$ the value $\phi$ for the tangle graph with variables $a$ and $b$ on the closing component (or edge). (Fig. 7)

Then from the discussion given in the reference [5] we can show that

$$
\phi(T, \boldsymbol{\alpha})_{b}^{a}=\lambda \delta_{a b} .
$$

The value of $\phi(T, \boldsymbol{\alpha})_{b}^{a}$ do not depend on $a$ or $b$.

For a colored graph $(F, \boldsymbol{\alpha})$ and a color $s$ of closing component (or edge), we define $\Phi$ by $\Phi(F, s, \boldsymbol{\alpha})=\lambda$ where $F, T, s$ are above and $\phi(T, \boldsymbol{\alpha})_{b}^{a}=\lambda \delta_{a b}$. By the basic relations given in $\S 6.2, \Phi$ is well-defined, i.e. $\Phi(F, s, \boldsymbol{\alpha})$ does not depend on a choice of $T$.

Further we have the next proposition, to obtain invariants which do not depend on $s$.

Proposition 6.3 [5] For a graph $F$ and its color $\boldsymbol{\alpha}=\left(p_{1}, \cdots, p_{n}\right)$, we have the next formula.

$$
\Phi(F, s, \boldsymbol{\alpha})\left(\left[p_{s} ; N-1\right]_{\epsilon} !\right)^{-1}=\Phi\left(F, s^{\prime}, \boldsymbol{\alpha}\right)\left(\left[p_{s^{\prime}} ; N-1\right]_{\epsilon} !\right)^{-1} .
$$

The proof of this proposition is equivalent to that given in Appendix $\mathrm{C}$ of the reference [5]. By this proposition we obtain the next definition.

Definition 6.4 For a trivalent colored oriented framed graph $(F, \boldsymbol{\alpha})$, we define an isotopy invariant $\hat{\Phi}$ of $(F, \boldsymbol{\alpha})$ by

$$
\hat{\Phi}(F, \boldsymbol{\alpha})=\Phi(F, s, \boldsymbol{\alpha})\left(\left[p_{s} ; N-1\right]_{\epsilon} !\right)^{-1} .
$$


Thus we obtain new invariants $\Phi(F, \boldsymbol{\alpha})$ of trivalent colored oriented framed graphs $(F, \boldsymbol{\alpha})$.

\section{Acknowledgement}

We would like to thank Prof. M. Wadati for encouragement. 


\section{A Appendix A}

We give derivation of the Clebsch-Gordan coefficients for infinite dimensional representations of $U_{q}(s l(2))$. We calculate the Clebsch-Gordan coefficients following Racah's approach [20]. For simplicity we denote $C\left(p_{1}, p_{2}, p_{1}+p_{2}-\right.$ $\left.n ; z_{1}, z_{2}, z\right)_{q}$ by $C\left(n ; z_{1}, z_{2}, z\right)$.

Applying $\Delta\left(X^{ \pm}\right)$on $\mid p_{1}, p_{2} ; p, z>\left(p=p_{1}+p_{2}-n\right)$, we have

$$
\begin{aligned}
& \sqrt{[z][2 p+1-z]} C\left(n ; z_{1}, z_{2}-1, z-1\right) \\
= & \sqrt{\left[z_{1}+1\right]\left[2 p_{1}-z_{1}\right]} q^{p_{2}-z_{2}+1} C\left(n ; z_{1}+1, z_{2}-1, z\right) \\
& +q^{-\left(p_{1}-z_{1}\right)} \sqrt{\left[z_{2}\right]\left[2 p_{2}+1-z_{2}\right]} C\left(n ; z_{1}, z_{2}, z\right), \\
& \sqrt{[z+1][2 p-z]} C\left(n ; z_{1}, z_{2}+1, z+1\right) \\
= & \sqrt{\left[z_{1}\right]\left[2 p_{1}+1-z_{1}\right]} q^{p_{2}-z_{2}-1} C\left(n ; z_{1}-1, z_{2}+1, z\right) \\
& +q^{-\left(p_{1}-z_{1}\right)} \sqrt{\left[z_{2}+1\right]\left[2 p_{2}-z_{2}\right]} C\left(n ; z_{1}, z_{2}, z\right) .
\end{aligned}
$$

We introduce $f\left(n ; z_{1}, z_{2}, z\right)$ by

$$
\begin{aligned}
& C\left(n ; z_{1}, z_{2}, z\right)=(-1)^{z_{1}} q^{\left(p_{1}-z_{1}\right)(p-z+1)} \\
& \times\left(\frac{\left[2 p_{1}-z_{1} ; n-z_{1}\right] !\left[2 p_{2}-z_{2} ; n-z_{2}\right] !}{[2 p ; z] !\left[z_{1}\right] !\left[z_{2}\right] ![z] !}\right)^{1 / 2} f\left(n ; z_{1}, z_{2}, z\right) .
\end{aligned}
$$

Then we have the following recurrence relations.

$$
\begin{aligned}
& f\left(n ; z_{1}, z_{2}+1\right)=-\left[z_{1}\right]\left[2 p_{1}+1-z_{1}\right] q^{2 p-2 z} f\left(n ; z_{1}-1, z_{2}+1, z\right) \\
& \quad+\left[2 p_{2}-z_{2}\right]\left[z_{2}+1\right] f\left(n ; z_{1}, z_{2}\right), \\
& {[z][2 p-z+1] q^{2\left(p_{1}-z_{1}\right)} f\left(n ; z_{1}, z_{2}-1\right)} \\
& =-f\left(n ; z_{1}+1, z_{2}-1, z\right)+f\left(n ; z_{1}, z_{2}, z\right) .
\end{aligned}
$$

Setting $z=0$ in (A.5) we have $f\left(n ; z_{1}, z_{2}, 0\right)=f\left(n ; z_{1}+1, z_{2}-1,0\right)$. Therefore we write $f\left(n ; z_{1}, z_{2}, 0\right)$ as $f_{n}$. From (A.4) we have

$$
f\left(n ; z_{1}, z_{2}, z\right)=f_{n}\left[z_{1}\right] !\left[z_{2}\right] ! \sum_{t}(-1)^{t} q^{t(2 p+1-z)}\left[\begin{array}{l}
z \\
t
\end{array}\right]
$$




$$
\times \frac{\left[2 p_{1}-z_{1}+t ; t\right] !\left[2 p_{2}-z_{2}+z-t ; z-t\right] !}{\left[z_{1}-t\right] !\left[z_{2}-z+t\right] !} .
$$

By using (B.3) it is easy to show that (A.6) satisfies the recurrence relation (A.4).

Now we consider $f_{n}$. If we assume the following expression of $C\left(n ; z_{1}, z_{2}, 0\right)$

$$
\begin{aligned}
C\left(n ; z_{1}, z_{2}, 0\right)= & (-1)^{z_{1}} q^{n(1-n) / 2-z_{1}(p+1)+n p_{2}} \\
& \times\left(\frac{[n] !\left[2 p_{1}-z_{1} ; n-z_{1}\right] !\left[2 p_{2}-z_{2} ; n-z_{2}\right] !}{[2 p+n+1 ; n] !\left[z_{1}\right] !\left[z_{2}\right] !}\right)^{1 / 2},(
\end{aligned}
$$

we can show the orthogonality relation

$$
\sum_{z_{1}} C\left(m ; z_{1}, n-z_{1}, n-m\right) C\left(n ; z_{1}, n-z_{1}, 0\right)=\delta_{n m}, \quad 0 \leq m \leq n .
$$

We prove (A.8) in the following. When $m=n$, we show (A.8) using (B.6) where $\alpha=2 p_{1}-n$ and $\beta=2 p_{2}-n$. When $m<n$, we prove (A.8) putting the expression (A.6) and (A.7) in the relation (A.8), transforming the variables $\left\{z_{1}, t\right\}$ into $\{t, \nu\}\left(z_{1}-t=\nu\right)$ and then taking the sum over $t$ using (B.4). Then we see that the sum vanishes.

From the relation (A.8) and the convention of phase factor, the expression (A.7) is shown. We obtain $f_{n}$ as follows.

$$
f_{n}=q^{n(1-n) / 2+n p_{2}-p_{1}(p+1)} \sqrt{\frac{[n] !}{[2 p+n+1 ; n] !}} .
$$

Thus we have the following expression for the Clebsch-Gordan coefficients.

$$
\begin{aligned}
C\left(n ; z_{1}, z_{2}, z\right)= & \delta\left(z_{1}+z_{2}-n, z\right) q^{n(1-n) / 2+n p_{2}-z_{1}(p+1)-\left(p_{1}-z_{1}\right) z} \\
& \times\left(\frac{\left[2 p_{1}-z_{1} ; n-z_{1}\right] !\left[2 p_{2}-z_{2} ; n-z_{2}\right] ![n] !\left[z_{1}\right] !\left[z_{2}\right] ![z] !}{[2 p+n+1 ; n] ![2 p ; z] !}\right)^{1 / 2} \\
& \sum_{t}(-1)^{t+z_{1}} q^{t(2 p+1-z)} \frac{\left[2 p_{1}-z_{1}+t ; t\right] !\left[2 p_{2}-z_{2}+z-t ; z-t\right] !}{[t] ![z-t] !\left[z_{1}-t\right] !\left[z_{2}-z+t\right] !} .
\end{aligned}
$$


Finally we transform the expression (A.10) of the Clebsch-Gordan coefficients into the symmetric one (3.10) given in the section 3 . We apply the formula (B.5) to the expression (A.10) $\left(\alpha=2 p_{1}-z_{1}, \beta=t, c=n-z_{1}+t\right)$, and replace the variable $k$ by $k=t-u$. We apply the formula (B.7) $\left(\alpha=2 p_{2}+z-z_{2}-u, s=t-u, a=z_{1}-u, b=z-u\right)$. Then we replace the variable $u$ by $\nu=z_{1}-u$. We thus obtain the symmetric expression (3.10).

We have considered only the case $p_{i} \in \mathbf{C}$. The Clebsch-Gordan coefficients for the other cases such as $\left(\mu_{1}, \mu_{2}\right)=\left(p_{1}, j_{2}\right)$ can be derived in the same way.

\section{B Appendix B}

We define $q$-analog of combinatorial for $m \geq k, m, k \in \mathbf{Z}_{\geq 0}$ and $\alpha \in \mathbf{C}$ as follows.

$$
\left[\begin{array}{c}
m \\
k
\end{array}\right]=\frac{[m] !}{[m-k] ![k] !}, \quad\left[\begin{array}{l}
\alpha \\
k
\end{array}\right]=\frac{[\alpha ; k] !}{[k] !} .
$$

(1) For $m, n \in \mathbf{Z}_{\geq 0}(n \geq m)$, we have the following useful formulas.

$$
\begin{aligned}
& \prod_{k=1}^{m}\left(1-z q^{2 k-2}\right)=\sum_{k}(-z)^{k} q^{k(m-1)}\left[\begin{array}{c}
m \\
k
\end{array}\right], \\
& {\left[\begin{array}{c}
n-1 \\
m
\end{array}\right]+\left[\begin{array}{c}
n-1 \\
m-1
\end{array}\right] q^{ \pm n}=\left[\begin{array}{c}
n \\
m
\end{array}\right] q^{ \pm m}} \\
& \sum_{k=0}^{n-m}(-1)^{k}\left[\begin{array}{c}
n-m \\
k
\end{array}\right] q^{k(n-m-1)}=\delta_{m n} .
\end{aligned}
$$

(2) For $\alpha, \beta \in \mathbf{C}$, and $m, n, a, b, c \in \mathbf{Z}_{\geq 0}$, we have the following.

$$
\begin{aligned}
& \sum_{k}\left[\begin{array}{c}
\alpha \\
c-k
\end{array}\right]\left[\begin{array}{l}
\beta \\
k
\end{array}\right] q^{ \pm k(\alpha+\beta)}=\left[\begin{array}{c}
\alpha+\beta \\
c
\end{array}\right] q^{ \pm c \beta}, \\
& \sum_{k}\left[\begin{array}{c}
\alpha+n-k \\
n-k
\end{array}\right]\left[\begin{array}{c}
\beta+k \\
k
\end{array}\right] q^{-k(\alpha+\beta+2)}=\left[\begin{array}{c}
\alpha+\beta+n+1 \\
n
\end{array}\right] q^{-n(1+\beta)},
\end{aligned}
$$




$$
\sum_{s}(-1)^{s}\left[\begin{array}{c}
\alpha-s \\
b-s
\end{array}\right]\left[\begin{array}{l}
a \\
s
\end{array}\right] q^{-s(-\alpha+a+b-1)}=\left[\begin{array}{c}
\alpha-a \\
b
\end{array}\right] q^{-a b} .
$$

We can derive $(\mathbb{B} .2)$ induction on $m$. We derive the relation $(\mathbb{B} .3)$ from (B.2). The relation (B.4) follows (B.2).

We show the relation (B.5) first for the case $\alpha=m_{1}, \beta=m_{2}\left(m_{1}, m_{2} \in\right.$ $\mathbf{Z}_{\geq 0}$ ) by using ( $\left.\overline{\mathrm{B} .2}\right)$. Since $m_{1}, m_{2}$ are arbitrary positive integers, and ( $\left.\overline{\mathrm{B} .5}\right)$ is equivalent to a polynomial relation in terms of $q^{\alpha}$ and $q^{\beta}$, the relation $(\mathbb{B . 5})$ holds also for complex parameters $\alpha, \beta$. From (B.5) we derive the relations (B.6) and (B.7) using the following

$$
\left[\begin{array}{l}
\alpha \\
n
\end{array}\right]=(-1)^{n}\left[\begin{array}{c}
-\alpha-1+n \\
n
\end{array}\right], \quad \text { for } \alpha \in \mathbf{C}, n \in \mathbf{Z}_{\geq 0}
$$




\section{References}

[1] Y. Akutsu and T. Deguchi, Phys. Rev. Lett. 67 (1991) 777.

[2] T. Deguchi and Y. Akutsu, J. Phys. Soc. Jpn. 60 (1991) 2559.

[3] T. Deguchi and Y. Akutsu, J. Phys. Soc. Jpn. 60 (1991) 4053.

[4] T. Deguchi, J. Phys. Soc. Jpn. 60 (1991) 3979.

[5] Y. Akutsu, T. Deguchi and T. Ohtsuki, Invariants of Colored Links, UTYO Math 91-24, preprint 1991 (to appear in Knot Theory and its Ramifications).

[6] T. Deguchi and Y. Akutsu, Colored braid matrices from infinite dimensional representations of $U_{q}(g)$, preprint 1991 .

[7] P. Roche and D. Arnaudon, Lett. Math. Phys. 17 (1989) 295-300.

[8] C. De Concini and V.G. Kac, Prog. Math. 92 (1990) pp. 471-506.

[9] C. De Concini, V.G. Kac and C. Procesi, Quantum Coadjoint Action, (Preprint di Matematica - n. 95, Scuola Normale Superiore, Pisa), preprint 1991.

[10] B.U. Felderhof, Physica 66 (1973) 279-297.

[11] C. Fan and F.Y. Wu: Phys. Rev. 179(1969) 650; Phys. rev. B2 (1970) 723.

[12] T.Deguchi, J. Phys. Soc. Jpn. 58 (1989) 3441-3444;

T.Deguchi and Y. Akutsu, J. Phys. A: Math. Gen. 23 (1990) 1861.

[13] C. L. Schultz: Phys. Rev. Lett. 46 (1981) 629. 
[14] J.H.H. Perk and C.L. Schultz, Phys. Lett. 84A (1981) 407, and in "NonLinear Integrable Systems, Classical Theory and Quantum Theory",ed. by M.Jimbo and T.Miwa, World Science, Singapore, 1981.

[15] P.P. Kulish, E.K. Sklyanin: J. Sov. Math. 19 (1982) 1596.

[16] L. Rozansky and H. Saleur, Quantum field theory for the multivariable Alexander Conway polynomial, YCTP-P20-91, preprint 1991;

L.H. Kauffman and H. Saleur, Fermions and Link Invariants, YCTPP21-91, preprint 1991.

[17] J. Murakami, The Free-Fermion Model in Presence of Field Related to the Quantum Group $U_{q}(\hat{s} l(2))$ of Affine Type and the Multi-Variable Alexander Polynomial of Links, RIMS-822, preprint 1991.

[18] J. Murakami, A state model for the multi-variable Alexander polynomial, preprint 1990.

[19] M. Couture, H.C. Lee, and N.C. Schmeing, in "Physics Geometry and Topology", ed. H.C. Lee, (Plenum Press, New York, 1990), 573-582.

[20] M.E. Rose, Elememtary Theory of Angular Momentum , John Wiley and Sons, Inc., New York, 1957.

[21] V.G.Drinfeld, "Quantum Groups" Proceedings of the ICM, Berkeley (1987) $798-820$.

[22] M. Jimbo, Lett. Math. Phys. 10 (1985) 63-69.

[23] A.N. Kirillov and N.Yu. Reshetikhin, in "Infinite Dimensional Lie Algebras and Groups", ed. V.G. Kac, World Scientific, 1989, p. 285.

[24] M. Nomura, J. Math. Phys. 30(1989) 2397-2405. 
[25] N. Yu Reshetikhin and V.G. Turaev, Invent. Math. (1991)

[26] D. Arnaudon, Phys. Lett. B268 (1991) 217.

[27] M. Jimbo, Commun. Math. Phys. 102 (1986) 537-547.

[28] V. Pasquier, Commun. Math. Phys. 118 (1988) 335.

[29] M. Nomura, J. Phys. Soc. Jpn.58 (1989) 2694.

[30] N. Yu. Reshetikhin and V.G. Turaev, Commun. Math. Phys. 127 (1990) $1-26$.

[31] Louis H. Kauffman, Trans. Amer. Math. Soc. 318 (1990) 317-371.

[32] Sergey Piunikhin, State sum models for trivalent knotted graph invariants using quantum groups $S L_{q}(2)$, preprint 1991. 


\section{Figure Captions}

Fig. 1

(a) Boltzmann weight $X_{\alpha \beta}(u)_{c d}^{a b}$ and (b) $R$ matrix $R_{p_{1} p_{2}}(u)_{b_{1} b_{2}}^{a_{1} a_{2}}$

Fig. 2

Boltzmann weight $w\left(a, b, c, d ; p_{1}, p_{2} ; u\right)$

Fig. 3

Trivalent vertex. The edges have colors $p_{1}, p_{2}, p$ and variables $z_{1}, z_{2}, z$

Fig. 4

(2,3)-tangle graph. Each segment in the graph has a color $p_{j}$.

Fig. 5

Relations D1 D7.

Fig. 6

We assign the follwoing weights to the diagrams.

(a) Identity diagram. $I_{b}^{a}=\delta_{a b},\left(I^{*}\right)_{b}^{a}=\delta_{a b}$

(b) Creation-annihilation diagrams.

$\left(U_{r}\right)_{a b}=q^{-p(N-1)} \epsilon^{-b} \delta_{a+b, N-1}$

$\left(U_{l}\right)_{a b}=q^{p(N-1)} \epsilon^{a} \delta_{a+b, N-1}$

$\left(\bar{U}_{r}\right)_{a b}=q^{p(N-1)} \epsilon^{a} \delta_{a+b, N-1}$

$\left(\bar{U}_{l}\right)_{a b}=q^{-p(N-1)} \epsilon^{-b} \delta_{a+b, N-1}$

(c) Braiding diagrams

$G\left(p_{1}, p_{2} ;+\right)_{c d}^{a b}$ and $G\left(p_{1}, p_{2} ;-\right)_{c d}^{a b}$

(d) Vertex diagrams.

We assign the Clebsch-Gordan coefficient $\left.C_{(} p_{1}, p_{2}, p ; z_{1}, z_{2}, z\right)$. both to the two vertex diagrams $V$ and $V^{\prime}$.

Fig. 7

The color $s$ of the closing edge (or component) is $p_{3}$. 